\title{
Cosmological constraints and phenomenology of a beyond-Horndeski model
}

\author{
Simone Peirone, ${ }^{1}$ Giampaolo Benevento, ${ }^{2,3,4}$ Noemi Frusciante, ${ }^{5}$ and Shinji Tsujikawa ${ }^{6}$ \\ ${ }^{1}$ Institute Lorentz, Leiden University, P.O. Box 9506, Leiden 2300 RA, The Netherlands \\ ${ }^{2}$ Dipartimento di Fisica e Astronomia "G. Galilei", Università degli Studi di Padova, \\ via Marzolo 8, I-35131 Padova, Italy \\ ${ }^{3}$ INFN, Sezione di Padova, via Marzolo 8, I-35131 Padova, Italy \\ ${ }^{4}$ Kavli Institute for Cosmological Physics, Department of Astronomy \& Astrophysics, \\ Enrico Fermi Institute, The University of Chicago, Chicago, Illinois 60637, USA \\ ${ }^{5}$ Instituto de Astrofísica e Ciências do Espaço, Faculdade de Ciências da Universidade de Lisboa, \\ Edificio C8, Campo Grande, P-1749016 Lisboa, Portugal \\ ${ }^{6}$ Department of Physics, Faculty of Science, Tokyo University of Science, \\ 1-3, Kagurazaka, Shinjuku-ku, Tokyo 162-8601, Japan
}

(Received 29 May 2019; published 10 September 2019)

\begin{abstract}
We study observational constraints on a specific dark energy model in the framework of GleyzesLanglois-Piazza-Vernizzi theories, which extends the Galileon ghost condensate (GGC) to the domain of beyond Horndeski theories. In this model, we show that the Planck cosmic microwave background (CMB) data, combined with datasets of baryon acoustic oscillations, supernovae type Ia, and redshift-space distortions, give the tight upper bound $\left|\alpha_{\mathrm{H}}^{(0)}\right| \leq \mathcal{O}\left(10^{-6}\right)$ on today's beyond-Horndeski (BH) parameter $\alpha_{\mathrm{H}}$. This is mostly attributed to the shift of $\mathrm{CMB}$ acoustic peaks induced by the early-time changes of cosmological background and perturbations arising from the dominance of $\alpha_{\mathrm{H}}$ in the dark energy density. In comparison to the $\Lambda$ cold dark matter $(\Lambda \mathrm{CDM})$ model, our $\mathrm{BH}$ model suppresses the large-scale integrated-Sachs-Wolfe tail of CMB temperature anisotropies due to the existence of cubic Galileons, and it modifies the small-scale $\mathrm{CMB}$ power spectrum because of the different background evolution. We find that the $\mathrm{BH}$ model considered fits the data better than $\Lambda \mathrm{CDM}$ according to the $\chi^{2}$ statistics, yet the deviance information criterion (DIC) slightly favors the latter. Given the fact that our BH model with $\alpha_{\mathrm{H}}=0$ (i.e., the GGC model) is favored over $\Lambda$ CDM even by the DIC, there are no particular signatures for the departure from Horndeski theories in current observations.
\end{abstract}

DOI: 10.1103/PhysRevD.100.063509

\section{INTRODUCTION}

Despite the tremendous progress of observational cosmology over the past two decades, the origin of today's acceleration of the Universe has not been identified yet. The standard concordance scenario is the $\Lambda \mathrm{CDM}$ model, in which the cosmological constant $\Lambda$ is the source for cosmic acceleration. In addition to the difficulty of naturally explaining the origin of $\Lambda$ from the vacuum energy [1-3], it is known that there are tensions between some datasets in the estimations of today's value of the Hubble constant $H_{0}=100 h \mathrm{~km} \mathrm{sec}^{-1} \mathrm{Mpc}^{-1}[4-8]$ and the amplitude $\sigma_{8}$ of the matter power spectrum on the scale of $8 h^{-1} \mathrm{Mpc}$ [9-13]. Such observational tensions along with the theoretical shortcoming of $\Lambda \mathrm{CDM}$ reinforce the idea to look for alternative models of dark energy [14-20].

Many extensions to the standard cosmological scenario include large-distance modifications of gravity due to an extra scalar degree of freedom (DOF), thus they are dubbed scalar-tensor theories [21]. Among those, the Horndeski class of theories [22] is the most general scheme with second-order equations of motion [23-25]. The latter feature ensures the absence of Ostrogradski instabilities, related to the existence of higher-order time derivatives.

It is possible to construct healthy theories beyond Horndeski gravity free from Ostrogradski instabilities. In Gleyzes-Langlois-Piazza-Vernizzi (GLPV) theories [26], for example, there are two extra Lagrangians beyond the Horndeski domain without increasing the extra propagating DOFs $[27,28]$. GLPV theories have several peculiar properties: the propagation speeds of matter and the scalar field are mixed [29-32], a partial breaking of the Vainshtein mechanism occurs inside astrophysical bodies [33-38], and a conical singularity can arise at the center of a spherically symmetric and static body $[39,40]$. We note that there exist also extensions of Horndeski theories containing higherorder spatial derivatives [41-43] (encompassing Horava gravity [44]) and degenerate higher-order scalar-tensor theories with one scalar propagating DOF [45-48].

The detection of the gravitational wave $(\mathrm{GW})$ signal GW170817 [49] accompanied by the gamma-ray burst event GRB170817A [50] shows that the speed of GWs $c_{t}$ is 
constrained to be in the range $-3 \times 10^{-15} \leq c_{t}-1 \leq 7 \times$ $10^{-16}[51]$ at the redshift $z \leq 0.009$, where we use the unit in which the speed of light $c$ is equivalent to 1 . The Horndeski Lagrangian, which gives the exact value $c_{t}=1$ without the tuning among functions, is of the form $L=G_{4}(\phi) R+$ $G_{2}(\phi, X)+G_{3}(\phi, X) \square \phi$, where $G_{4}$ is a function of the scalar field $\phi, R$ is the Ricci scalar, and $G_{2,3}$ depend on both $\phi$ and $X=\partial^{\mu} \phi \partial_{\mu} \phi$ [52-57]. There are also dark energy models in which the GW speed consistent with the above observational bound of $c_{t}$ can be realized [58-60]. In GLPV theories with the $X$ dependence in $G_{4}$, it is also possible to realize $c_{t}=1$ by the existence of an additional quartic Lagrangian beyond the Horndeski domain [61].

In addition to the bound on $c_{t}$, the absence of the decay of GWs into dark energy at LIGO/Virgo frequencies $(f \sim$ $100 \mathrm{~Hz}$ ) may imply that the parameter $\alpha_{\mathrm{H}}$ characterizing the deviation from Horndeski theories is constrained to be very tiny for the scalar sound speed $c_{s}$ different from 1 , typically of order $\left|\alpha_{\mathrm{H}}\right| \lesssim 10^{-10}$ today [62]. If we literally use this bound, there is little room left for dark energy models in beyond-Horndeski theories $[63,64]$. If $c_{s}$ is equivalent to 1 , the decay of GWs into dark energy is forbidden. However, it was argued in Ref. [62] that power-law divergent terms would appear, leading to the conclusion that the operator accompanying $\alpha_{\mathrm{H}}$ must be suppressed as well [62].

We note that the LIGO/Virgo frequencies are close to those of the typical strong coupling scale or cutoff $\Lambda_{c}$ of dark energy models containing derivative field selfinteractions [65]. Around this cutoff scale, we cannot exclude the possibility that some ultraviolet (UV) effects come into play to recover the propagation and property of GWs similar to those in general relativity (GR). If this kind of UV completion occurs around the frequency $f \sim 100 \mathrm{~Hz}$, the aforementioned bounds on $c_{t}$ and $\alpha_{\mathrm{H}}$ are not applied to the effective field theory of dark energy exploited to describe the cosmological dynamics much below the energy scale $\Lambda_{c}$. Future space-based missions, such as LISA [66], are sensitive to much lower frequencies ( $f \sim 10^{-3} \mathrm{~Hz}$ ), so they will offer further valuable information on the properties of GWs with different frequencies.

In GLPV theories, there are constraints on the parameter $\alpha_{\mathrm{H}}$ arising from the modifications to gravitational interactions inside astrophysical objects. For example, the consistency of the minimum mass for hydrogen burning in stars with the red dwarf of lowest mass shows that $\left|\alpha_{\mathrm{H}}\right|$ is at most of order $0.1[35,36,67,68]$. By using $\mathrm{x}$-ray and lensing profiles of galaxy clusters, similar bounds on $\alpha_{\mathrm{H}}$ were obtained in Ref. [37]. From the orbital period of the Hulse-Taylor binary pulsar PSR B1913 + 1, the upper bound of $\left|\alpha_{\mathrm{H}}\right|$ is of order $10^{-3}$ [69]. Cosmological constraints on $\alpha_{\mathrm{H}}$ were derived by using particular parametric forms of dimensionless quantities appearing in the effective field theory of dark energy to describe their evolution. In this case, the constraints from $\mathrm{CMB}$ and large-scale structure data on $\left|\alpha_{\mathrm{H}}\right|$ are of order $\mathcal{O}(1)$ [70].
In this paper, we place observational bounds on the beyond-Horndeski $(\mathrm{BH})$ dark energy model proposed in Ref. [61] and study how the parameter $\alpha_{\mathrm{H}}$ is constrained from the cosmological datasets of $\mathrm{CMB}$ temperature anisotropies, baryon acoustic oscillations (BAO), supernovae type Ia (SN Ia), and redshift-space distortions (RSDs). In the limit $\alpha_{\mathrm{H}} \rightarrow 0$, the model reduces to the Galileon ghost condensate (GGC) in Horndeski theories. The recent analysis of Ref. [71] reveals that the GGC model is observationally favored over $\Lambda \mathrm{CDM}$ according to several information criteria. We will investigate whether or not this property persists for the BH dark energy model $\left(\alpha_{\mathrm{H}} \neq 0\right)$ of Ref. [61]. For the likelihood analysis, we will use the publicly available effective field theory for CAMB (EFTCAMB) code ${ }^{1}$ $[72,73]$. In our investigation the gravitational theory is completely determined by a covariant action, while the analysis in Ref. [70] follows a parametrized approach to GLPV theories. In this respect, the two cosmological models considered are completely different and the constraint on $\alpha_{\mathrm{H}}$ obtained in this paper cannot be straightforwardly compared to the results in Ref. [70].

The paper is organized as follows. In Sec. II, we briefly review the basics of the BH dark energy model introduced in Ref. [61]. In Sec. III, we show how this model can be implemented in the EFT formulation and derive the background equations of motion together with theoretically consistent conditions. In Sec. IV, we discuss the evolution of cosmological perturbations in the presence of matter perfect fluids and investigate the impact of our model on observable quantities. In Sec. V, we present the MonteCarlo-Markov-chain (MCMC) constraints on model parameters and compute several information criteria to discuss whether the BH model is favored over the $\Lambda \mathrm{CDM}$ model. Finally, we conclude in Sec. VI.

\section{DARK ENERGY MODEL IN GLPV THEORIES}

The dark energy model proposed in Ref. [61] belongs to the quartic-order GLPV theories given by the action

$$
\mathcal{S}=\int \mathrm{d}^{4} x \sqrt{-g} \sum_{i=2}^{4} L_{i}+\mathcal{S}_{M}\left[g_{\mu \nu}, \chi_{M}\right]
$$

where $g$ is the determinant of metric tensor $g_{\mu \nu}, \mathcal{S}_{M}$ is the matter action for all matter fields $\chi_{M}$, and the Lagrangians $L_{2,3,4}$ are defined by

$$
\begin{aligned}
L_{2}= & G_{2}(\phi, X), \\
L_{3}= & G_{3}(\phi, X) \square \phi, \\
L_{4}= & G_{4}(\phi, X) R-2 G_{4, X}(\phi, X)\left[(\square \phi)^{2}-\phi^{\mu \nu} \phi_{\mu \nu}\right] \\
& +F_{4}(\phi, X) \epsilon_{\sigma}^{\mu \nu \rho} \epsilon^{\mu^{\prime} \nu^{\prime} \rho^{\prime} \sigma} \phi_{\mu^{\prime}} \phi_{\mu} \phi_{\nu \nu^{\prime}} \phi_{\rho \rho^{\prime}},
\end{aligned}
$$

\footnotetext{
${ }^{1}$ Web page: http://www.eftcamb.org.
} 
where $G_{2,3,4}$ and $F_{4}$ are functions of the scalar field $\phi$ and $X=\nabla^{\mu} \phi \nabla_{\mu} \phi, R$ is the Ricci scalar, and $\epsilon^{\mu \nu \rho \sigma}$ is the totally antisymmetric Levi-Civita tensor satisfying the normalization $\epsilon^{\mu \nu \rho \sigma} \epsilon_{\mu \nu \rho \sigma}=+4$ !. We also define $G_{i, X} \equiv \partial G_{i} / \partial X$ and use the notations $\phi_{\mu}=\nabla_{\mu} \phi$ and $\phi_{\mu \nu}=\nabla_{\nu} \nabla_{\mu} \phi$ for the covariant derivative operator $\nabla_{\mu}$. We assume that the matter fields $\chi_{M}$ are minimally coupled to gravity.

The last term containing $F_{4}(\phi, X)$ in $L_{4}$ arises beyond the domain of Horndeski theories [26]. The deviation from Horndeski theories can be quantified by the parameter

$$
\alpha_{\mathrm{H}}=-\frac{X^{2} F_{4}}{G_{4}-2 X G_{4, X}+X^{2} F_{4}},
$$

which does not vanish for $F_{4} \neq 0$. The line element containing intrinsic tensor perturbations $h_{i j}$ on the flat Friedmann-Lemaître-Robertson-Walker (FLRW) spacetime is given by

$$
\mathrm{d} s^{2}=-\mathrm{d} t^{2}+a^{2}(t)\left(\delta_{i j}+h_{i j}\right) \mathrm{d} x^{i} \mathrm{~d} x^{j},
$$

where $a(t)$ is the time-dependent scale factor, and $h_{i j}$ satisfies the transverse and traceless conditions $\left(\nabla^{j} h_{i j}=0\right.$ and $h_{i}{ }^{i}=0$ ). The propagation speed squared of tensor perturbations is $[26,29,30]$

$$
c_{t}^{2}=\frac{G_{4}}{G_{4}-2 X G_{4, X}+X^{2} F_{4}} .
$$

In quartic-order Horndeski theories $\left(F_{4}=0\right)$, the $X$ dependence in $G_{4}$ leads to the difference of $c_{t}^{2}$ from 1 . In GLPV theories, it is possible to realize $c_{t}^{2}=1$ for the function

$$
F_{4}=\frac{2 G_{4, X}}{X}
$$

under which $\alpha_{\mathrm{H}}=-2 X G_{4, X} / G_{4}$.

In this paper, we will study observational constraints on the model proposed in Ref. [61]. This is characterized by the following functions:

$$
\begin{aligned}
& G_{2}=a_{1} X+a_{2} X^{2}, \quad G_{3}=3 a_{3} X, \\
& G_{4}=\frac{m_{0}^{2}}{2}-a_{4} X^{2},
\end{aligned}
$$

where $m_{0}$ and $a_{1,2,3,4}$ are constants. This beyond-Horndeski model, hereafter $\mathrm{BH}$, satisfies the condition (2.6), and hence $c_{t}^{2}=1$. When $a_{4}=0, \mathrm{BH}$ recovers the GGC model studied recently in Ref. [71]. Taking the limits $a_{2} \rightarrow 0$ and $a_{3} \rightarrow 0$, GGC recovers the cubic covariant Galileon [74,75] and ghost condensate [76], respectively.

The BH model allows for the existence of selfaccelerating de Sitter solutions finally approaching constant values of $X$. Before approaching the de Sitter attractor, the dark energy equation of state $w_{\mathrm{DE}}$ can exhibit a phantom behavior (i.e., $w_{\mathrm{DE}}<-1$ ) without the appearance of ghosts [61]. The cubic covariant Galileon gives rise to the tracker solution with $w_{\mathrm{DE}}=-2$ in the matter era [77], but this evolution is incompatible with the joint data analysis of $\mathrm{CMB}, \mathrm{BAO}$, and SN Ia [78]. On the other hand, in both BH and GGC, the $a_{2} X^{2}$ term works to prevent for approaching the tracker, so that $-2<w_{\mathrm{DE}}<-1$ in the matter era. This behavior of $w_{\mathrm{DE}}$ is consistent with the recent observational datasets of $\mathrm{CMB}, \mathrm{BAO}$, and $\mathrm{SN}$ Ia [71].

The $\mathrm{BH}$ model leads to the evolution of cosmological perturbations different from that in GR. The late-time modification to the cosmic growth rate arises mostly from the cubic Galileon term $3 a_{3} X \square \phi$ [61,79]. In GGC, the combined effect of $3 a_{3} X \square \phi$ and $a_{2} X^{2}$ can suppress the power spectrum of large-scale CMB temperature anisotropies, so that the model shows a better compatibility with the Planck data with respect to the $\Lambda$ cold dark matter $(\Lambda \mathrm{CDM})$ [71]. It remains to be seen whether the similar property also holds for the BH model with $a_{4} \neq 0$, which we will address in this paper.

\section{METHODOLOGY}

In this section, we discuss the evolution of the background and linear scalar perturbations in the $\mathrm{BH}$ model. We make use of the EFTCAMB/EFTCosmoMC codes [72,73], in which the EFT of dark energy and modified gravity [8084] is implemented into CAMB/CosmoMC [85,86]. The EFT framework enables one to deal with any dark energy and modified gravity model with one scalar propagating DOF $\phi$ in a unified and model-independent manner.

The EFT of dark energy is based on the $3+1$ ArnowittDeser-Misner (ADM) decomposition of spacetime [87] given by the line element

$$
\mathrm{d} s^{2}=-N^{2} \mathrm{~d} t^{2}+h_{i j}\left(\mathrm{~d} x^{i}+N^{i} \mathrm{~d} t\right)\left(\mathrm{d} x^{j}+N^{j} \mathrm{~d} t\right),
$$

where $N$ is the lapse, $N^{i}$ is the shift vector, and $h_{i j}$ is the three-dimensional metric. A unit vector orthogonal to the constant time hypersurface $\Sigma_{t}$ is given by $n_{\mu}=$ $N \nabla_{\mu} t=(N, 0,0,0)$. The extrinsic curvature is defined by $K_{i j}=h_{i}^{k} \nabla_{k} n_{j}$. The internal geometry of $\Sigma_{t}$ is quantified by the three-dimensional Ricci tensor $\mathcal{R}_{i j}={ }^{(3)} R_{i j}$ associated with the metric $h_{i j}$.

On the flat FLRW background, we consider the line element containing three scalar metric perturbations $\delta N, \psi$, and $\zeta$, as

$$
\begin{aligned}
\mathrm{d} s^{2}= & -(1+2 \delta N) \mathrm{d} t^{2}+2 \partial_{i} \psi \mathrm{d} t \mathrm{~d} x^{i} \\
& +a^{2}(t)(1+2 \zeta) \delta_{i j} \mathrm{~d} x^{i} \mathrm{~d} x^{j},
\end{aligned}
$$

where $\partial_{i} \equiv \partial / \partial x^{i}$. We also choose the unitary gauge in which the perturbation $\delta \phi$ of the scalar field $\phi$ vanishes. 
Then, the perturbations of extrinsic and intrinsic curvatures are expressed as $[29,42,83,84]$

$$
\begin{gathered}
\delta K_{i j}=a^{2}(H \delta N-2 H \zeta-\dot{\zeta}) \delta_{i j}+\partial_{i} \partial_{j} \psi, \\
\delta \mathcal{R}_{i j}=-\delta_{i j} \partial^{2} \zeta-\partial_{i} \partial_{j} \zeta
\end{gathered}
$$

where $\partial^{2} \equiv \delta^{k l} \partial_{k} \partial_{l}$, and $H=\dot{a} / a$ is the Hubble expansion rate, and a dot represents a derivative with respect to $t$. The perturbations of traces $K \equiv K^{i}{ }_{i}$ and $\mathcal{R} \equiv \mathcal{R}^{i}{ }_{i}$ are denoted as $\delta K$ and $\delta \mathcal{R}$, respectively, with $\delta g^{00}=2 \delta N$.

In the ADM language, the Lagrangian of GLPV theories depends on the scalar quantities $N, K, K_{i j} K^{i j}, \mathcal{R}, K_{i j} \mathcal{R}^{i j}$, and $t$ [83]. Expanding the corresponding action up to second order in scalar perturbations of those quantities, it follows that

$$
\begin{aligned}
\mathcal{S}= & \int \mathrm{d}^{4} x \sqrt{-g} m_{0}^{2}\left\{\frac{1}{2}[1+\Omega(a)] R+\frac{\Lambda(a)}{m_{0}^{2}}-\frac{c(a)}{m_{0}^{2}} \delta g^{00}\right. \\
& +H_{0}^{2} \frac{\gamma_{1}(a)}{2}\left(\delta g^{00}\right)^{2}-H_{0} \frac{\gamma_{2}(a)}{2} \delta g^{00} \delta K \\
& -H_{0}^{2} \frac{\gamma_{3}(a)}{2}(\delta K)^{2}-H_{0}^{2} \frac{\gamma_{4}(a)}{2} \delta K_{j}^{i} \delta K_{i}^{j} \\
& \left.+\frac{\gamma_{5}(a)}{2} \delta g^{00} \delta \mathcal{R}\right\}+\mathcal{S}_{M}\left[g_{\mu \nu}, \chi_{M}\right]
\end{aligned}
$$

where $m_{0}$ is a constant having a dimension of mass, and $\Omega$, $\Lambda, c, \gamma_{i}$ are called EFT functions that depend on the background scale factor $a$. The explicit relations between those EFT functions and the functions $G_{2,3,4}, F_{4}$ in the action (2.1) are given in Ref. [88].

The first three variables $\Omega, \Lambda, c$ determine both the background evolution and linear perturbations, whereas the functions $\gamma_{i}$ solely appear at the level of linear perturbations. For the matter action $\mathcal{S}_{M}$, we take dark matter and baryons (background density $\rho_{m}$ and vanishing pressure) and radiation (background density $\rho_{r}$ and pressure $P_{r}=\rho_{r} / 3$ ) into account. Then, the background equations are expressed as $[80,81]$

$$
\begin{gathered}
3 m_{0}^{2} H^{2}=\rho_{\mathrm{DE}}+\rho_{m}+\rho_{r}, \\
-m_{0}^{2}\left(2 \dot{H}+3 H^{2}\right)=P_{\mathrm{DE}}+P_{r},
\end{gathered}
$$

where

$$
\begin{gathered}
\rho_{\mathrm{DE}}=2 c-\Lambda-3 m_{0}^{2} H(\dot{\Omega}+H \Omega), \\
P_{\mathrm{DE}}=\Lambda+m_{0}^{2}\left[\ddot{\Omega}+2 H \dot{\Omega}+\Omega\left(2 \dot{H}+3 H^{2}\right)\right] .
\end{gathered}
$$

The density $\rho_{\mathrm{DE}}$ and pressure $P_{\mathrm{DE}}$ of dark energy obey the continuity equation

$$
\dot{\rho}_{\mathrm{DE}}+3 H\left(\rho_{\mathrm{DE}}+P_{\mathrm{DE}}\right)=0 .
$$

In GLPV theories, there is the specific relation $\gamma_{3}=-\gamma_{4}$. If we restrict the theories to those satisfying $c_{t}^{2}=1$, it follows that $\gamma_{4}=0$. Then, the model given by the functions (2.7) corresponds to the coefficients

$$
\gamma_{3}=0, \quad \gamma_{4}=0,
$$

so that we are left with three functions $\gamma_{1}, \gamma_{2}, \gamma_{5}$ at the level of linear perturbations.

To study the cosmological evolution of our model in EFTCAMB, we first solve the background equations of motion and then map to the EFT functions according to the procedure given in Refs. [43,80-84,88].

\section{A. Background equations in the $\mathrm{BH}$ model}

For the model (2.7), the background equations are given by Eqs. (3.6) and (3.7), with

$$
\Omega=-\frac{2 a_{4} \dot{\phi}^{4}}{m_{0}^{2}},
$$

and

$$
\begin{aligned}
& \rho_{\mathrm{DE}}=-a_{1} \dot{\phi}^{2}+3 a_{2} \dot{\phi}^{4}+18 a_{3} H \dot{\phi}^{3}+30 a_{4} H^{2} \dot{\phi}^{4}, \\
& P_{\mathrm{DE}}=-a_{1} \dot{\phi}^{2}+a_{2} \dot{\phi}^{4}-6 a_{3} \dot{\phi}^{2} \ddot{\phi} \\
&-2 a_{4} \dot{\phi}^{3}\left[8 H \ddot{\phi}+\dot{\phi}\left(2 \dot{H}+3 H^{2}\right)\right] .
\end{aligned}
$$

The parameters $c$ and $\Lambda$ in Eqs. (3.8) and (3.9) can be expressed in terms of quantities on the right-hand sides of Eqs. (3.13) and (3.14). Following Ref. [61], we define the dimensionless variables (density parameters):

$$
\begin{aligned}
& x_{1}=-\frac{a_{1} \dot{\phi}^{2}}{3 m_{0}^{2} H^{2}}, \quad x_{2}=\frac{a_{2} \dot{\phi}^{4}}{m_{0}^{2} H^{2}}, \\
& x_{3}=\frac{6 a_{3} \dot{\phi}^{3}}{m_{0}^{2} H}, \quad x_{4}=\frac{10 a_{4} \dot{\phi}^{4}}{m_{0}^{2}},
\end{aligned}
$$

and

$\Omega_{\mathrm{DE}}=\frac{\rho_{\mathrm{DE}}}{3 m_{0}^{2} H^{2}}, \quad \Omega_{m}=\frac{\rho_{m}}{3 m_{0}^{2} H^{2}}, \quad \Omega_{r}=\frac{\rho_{r}}{3 m_{0}^{2} H^{2}}$.

From Eq. (3.6), we have

$$
\Omega_{m}=1-\Omega_{\mathrm{DE}}-\Omega_{r},
$$

where the dark energy density parameter is given by

$$
\Omega_{\mathrm{DE}}=x_{1}+x_{2}+x_{3}+x_{4} \text {. }
$$


In terms of $x_{4}$, the deviation parameter (2.3) from Horndeski theories is expressed as

$$
\alpha_{\mathrm{H}}=\frac{4 x_{4}}{5-x_{4}}
$$

and hence $\alpha_{\mathrm{H}}$ is of the same order as $x_{4}$ for $\left|x_{4}\right| \leq 1$.

The variables $x_{1,2,3,4}$ and $\Omega_{r}$ are known by solving the ordinary differential equations

$$
\begin{gathered}
x_{1}^{\prime}=2 x_{1}\left(\epsilon_{\phi}-h\right), \\
x_{2}^{\prime}=2 x_{2}\left(2 \epsilon_{\phi}-h\right), \\
x_{3}^{\prime}=x_{3}\left(3 \epsilon_{\phi}-h\right), \\
x_{4}^{\prime}=4 x_{4} \epsilon_{\phi}, \\
\Omega_{r}^{\prime}=-2 \Omega_{r}(2+h),
\end{gathered}
$$

where a prime denotes the derivative with respect to $\mathcal{N}=\ln (a)$. On using Eqs. (3.6) and (3.7), it follows that

$$
\begin{aligned}
\epsilon_{\phi} \equiv & \frac{\ddot{\phi}}{H \dot{\phi}} \\
= & -\frac{1}{q_{s}}\left[20\left(3 x_{1}+2 x_{2}\right)-5 x_{3}\left(3 x_{1}+x_{2}+\Omega_{r}-3\right)\right. \\
& \left.-x_{4}\left(36 x_{1}+16 x_{2}+3 x_{3}+8 \Omega_{r}\right)\right], \\
h \equiv & \frac{\dot{H}}{H^{2}} \\
= & -\frac{1}{q_{s}}\left[10\left(3 x_{1}+x_{2}+\Omega_{r}+3\right)\left(x_{1}+2 x_{2}\right)\right. \\
& +10 x_{3}\left(6 x_{1}+3 x_{2}+\Omega_{r}+3\right)+15 x_{3}^{2} \\
& \left.+x_{4}\left(78 x_{1}+32 x_{2}+30 x_{3}+12 \Omega_{r}+36\right)+12 x_{4}^{2}\right],
\end{aligned}
$$

with

$$
\begin{aligned}
q_{s} \equiv & 20\left(x_{1}+2 x_{2}+x_{3}\right)+4 x_{4}\left(6-x_{1}-2 x_{2}+3 x_{3}\right) \\
& +5 x_{3}^{2}+8 x_{4}^{2} .
\end{aligned}
$$

For a given set of initial conditions $x_{1,2,3,4}$ and $\Omega_{r}$, we can solve Eqs. (3.20)-(3.24) to determine the evolution of density parameters as well as $\phi$ and $H$. Practically, we start to solve the above dynamical system at redshift $z_{s}=1.5 \times$ $10^{5}$ and iteratively scan over initial conditions leading to the viable cosmology satisfying the constraint (3.17) today $(z=0)$. Additionally, evaluating Eq. (3.18) at present time, we can eliminate one model parameter, for example $x_{2}^{(0)}$, as $x_{2}^{(0)}=\Omega_{\mathrm{DE}}^{(0)}-x_{1}^{(0)}-x_{3}^{(0)}-x_{4}^{(0)}$, where “(0)” represents today's quantities.

\section{B. Mapping}

To study the evolution of scalar perturbations and observational constraints on dark energy models in EFTCAMB, it is convenient to use the mapping between EFT functions and model parameters in BH. In Sec. III A, we already discussed the mapping of the background quantities $\Omega, \Lambda$ and $c$. The functions $\gamma_{1,2,5}$, which are associated with scalar perturbations, are given by

$$
\begin{gathered}
\gamma_{1}=\frac{H^{2}}{H_{0}^{2}}\left[\frac{1}{20}\left(24 x_{4}-h x_{4}^{\prime}+3 x_{4}^{\prime}-x_{4}^{\prime \prime}\right)\right. \\
\left.+2 x_{2}+\frac{1}{12}\left\{(h+9) x_{3}+x_{3}^{\prime}\right\}\right], \\
\gamma_{2}=\frac{H}{H_{0}}\left[\frac{1}{5}\left(x_{4}^{\prime}-8 x_{4}\right)-x_{3}\right], \\
\gamma_{5}=\frac{2}{5} x_{4} .
\end{gathered}
$$

The expressions of these EFT functions allow us to draw already some insight about the contributions of each $x_{i}$ to the dynamics of linear perturbations. In general, the variable $\gamma_{1}$ cannot be well constrained by data being its contribution to the observables below the cosmic variance [89]. The main modification to the evolution of perturbations compared to GR arises from $\gamma_{2}$ and $\gamma_{5}$, which are mostly affected by $x_{3}$ and $x_{4}$. The variables $x_{1}$ and $x_{2}$ contribute to the perturbation dynamics through the Hubble expansion rate $H$ in $\gamma_{2}$.

\section{Viability constraints}

There are theoretically consistent conditions under which the perturbations are not plagued by the appearance of ghosts and Laplacian instabilities in the small-scale limit. For the BH model (2.7), the conditions for the absence of ghosts in tensor and scalar sectors are given, respectively, by [61]

$$
\begin{gathered}
Q_{t}=\frac{5-x_{4}}{10} m_{0}^{2}>0, \\
Q_{s}=\frac{3\left(5-x_{4}\right) q_{s}}{25\left(x_{3}+2 x_{4}-2\right)^{2}} m_{0}^{2}>0,
\end{gathered}
$$

where $q_{s}$ is defined in Eq. (3.25). Then, we have the following constraints:

$$
x_{4}<5, \quad q_{s}>0 .
$$

The $\mathrm{BH}$ model has the property $c_{t}^{2}=1$, so there is no Laplacian instability for tensor perturbations. We note that the reduced Planck mass $M_{\mathrm{pl}}$ is related to $m_{0}$ according to the relation $M_{\mathrm{pl}}^{2}=m_{0}^{2}\left(1+\Omega_{0}\right)$ in the local environment 
with screened fifth forces [33], where $\Omega_{0}$ is today's value of $\Omega$. Then, the Newton gravitational constant $G_{\mathrm{N}}$ is given by

$$
G_{\mathrm{N}}=\frac{1}{8 \pi M_{\mathrm{pl}}^{2}}=\frac{1}{8 \pi m_{0}^{2}}\left(1-\frac{x_{4}^{(0)}}{5}\right)^{-1},
$$

which is positive under the absence of tensor ghosts.

For scalar perturbations, there are three propagation speed squares $c_{s}^{2}, \tilde{c}_{r}^{2}$, and $\tilde{c}_{m}^{2}$ associated with the scalar field $\phi$, radiation, and nonrelativistic matter, respectively. In Horndeski theories, they are not coupled to each other, so that the propagation speed squares of radiation and nonrelativistic matter are given, respectively, by $c_{r}^{2}=1 / 3$ and $c_{m}^{2}=+0$. In GLPV theories, they are generally mixed with each other, apart from $\tilde{c}_{m}^{2}$ (which has the value $\tilde{c}_{m}^{2}=+0$ ) [26,29-32]. Then, the Laplacian instabilities of scalar perturbations can be avoided under the two conditions

$$
\begin{aligned}
& c_{s}^{2}=\frac{1}{2}\left(c_{r}^{2}+c_{\mathrm{H}}^{2}-\beta_{\mathrm{H}}-\gamma_{\mathrm{H}}\right)>0, \\
& \tilde{c}_{r}^{2}=\frac{1}{2}\left(c_{r}^{2}+c_{\mathrm{H}}^{2}-\beta_{\mathrm{H}}+\gamma_{\mathrm{H}}\right)>0,
\end{aligned}
$$

where

$$
\begin{aligned}
c_{\mathrm{H}}^{2} & =\frac{2}{Q_{s}}\left[\dot{\mathcal{M}}+H \mathcal{M}-Q_{t}-\frac{3 \rho_{m}+4 \rho_{r}}{12 H^{2}\left(1+\alpha_{\mathrm{B}}\right)^{2}}\right], \\
\beta_{r} & =\frac{4 \alpha_{\mathrm{H}} \rho_{r}}{3 Q_{s} H^{2}\left(1+\alpha_{\mathrm{B}}\right)^{2}}, \quad \beta_{m}=\frac{\alpha_{\mathrm{H}} \rho_{m}}{Q_{s} H^{2}\left(1+\alpha_{\mathrm{B}}\right)^{2}}, \\
\mathcal{M} & =\frac{Q_{t}\left(1+\alpha_{\mathrm{H}}\right)}{H\left(1+\alpha_{\mathrm{B}}\right)}, \quad \beta_{\mathrm{H}}=\beta_{r}+\beta_{m}, \quad \alpha_{\mathrm{B}}=-\frac{5 x_{3}+8 x_{4}}{2\left(5-x_{4}\right)}, \\
\gamma_{\mathrm{H}} & =\sqrt{\left(c_{r}^{2}-c_{\mathrm{H}}^{2}+\beta_{\mathrm{H}}\right)^{2}+2 c_{r}^{2} \alpha_{\mathrm{H}} \beta_{r}} .
\end{aligned}
$$

When $\left|\alpha_{\mathrm{H}}\right| \ll 1$ we have $c_{s}^{2} \simeq c_{\mathrm{H}}^{2}-\beta_{\mathrm{H}}$ and $\tilde{c}_{r}^{2} \simeq c_{r}^{2}=1 / 3$, so the second stability condition (3.34) is satisfied.

There are also constraints on today's parameter $\alpha_{\mathrm{H}}^{(0)}$ (or equivalently, $x_{4}^{(0)}$ ) from massive astrophysical objects $[35,36,69]$. Among those constraints, the orbital period of Hulse-Taylor binary pulsar gives the tightest bound $-0.0031 \leq x_{4}^{(0)} \leq 0.0094[61,69]$. If we literally use the bound arising from the absence of the GW decay into dark energy at LIGO/Virgo frequencies, the parameter $\alpha_{\mathrm{H}}^{(0)}$ should be less than the order of $10^{-10}$ [62]. As we mentioned in Introduction, it is still a matter of debate whether the EFT of dark energy is valid around the frequency $f \sim 100 \mathrm{~Hz}$ [65]. In this paper, we will not impose such a bound and independently test how the cosmological observations place the upper limit of $x_{4}^{(0)}$.

In Fig. 1, we show the physically viable parameter space (blue colored region) for the initial conditions $x_{1}^{(s)}, x_{2}^{(s)}, x_{3}^{(s)}$,
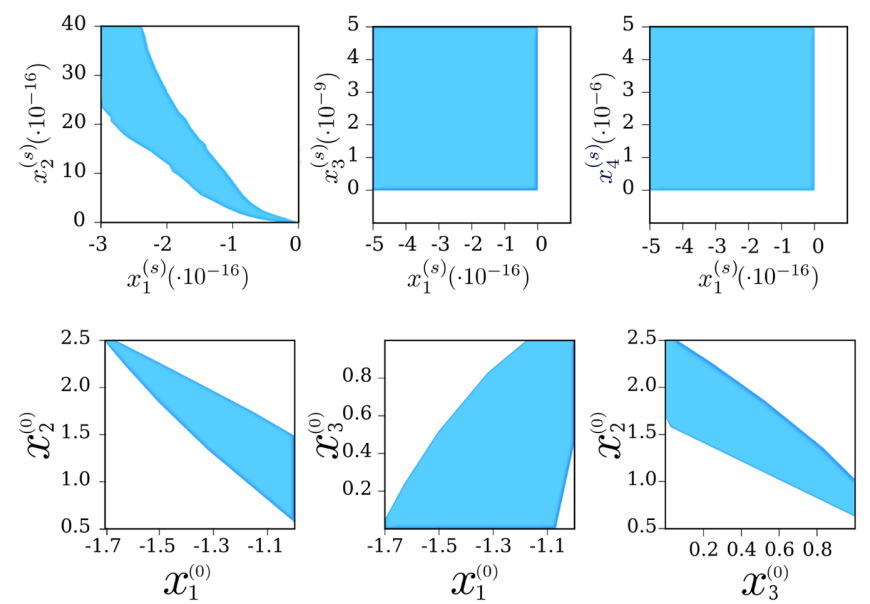

FIG. 1. The viable parameter space (in blue) for the initial values $x_{1}^{(s)}, x_{2}^{(s)}, x_{3}^{(s)}$ and $x_{4}^{(s)}$ at the redshift $z_{s}=1.5 \times 10^{5}$ (top panel) and today's parameters $x_{1}^{(0)}, x_{2}^{(0)}$ and $x_{3}^{(0)}$ (bottom panel). In these parameter spaces, there are neither ghosts nor Laplacian instabilities.

$x_{4}^{(s)} \quad$ (at redshift $z_{s}=1.5 \times 10^{5}$ ) and today's values $x_{1}^{(0)}, x_{2}^{(0)}, x_{3}^{(0)}$ (at redshift $z=0$ ). We find that $x_{1}^{(0)}$ is negative, while $x_{2}^{(0)}$ and $x_{3}^{(0)}$ are positive. We note that the ghost condensate model [76] has a de Sitter solution satisfying $x_{1}<0$ and $x_{2}>0$. The Galileon term $x_{3}$ modifies the cosmological dynamics of ghost condensate, but there is also a de Sitter attractor characterized by $x_{1}<0, x_{2}>0$, and $x_{3}>0$ [61]. As we see in Fig. 1, the parameter $x_{3}^{(0)}$ is not well constrained from the theoretically viable conditions alone.

The parameter space of the variable $x_{4}^{(0)}$ is not shown in Fig. 1, but it is in the range $\left|x_{4}^{(0)}\right| \ll 1$ to satisfy all the theoretically consistent conditions. As $x_{4}^{(0)}$ approaches the order 1 , the scalar perturbation is typically prone to the Laplacian instability associated with the negative value of $c_{s}^{2}$ [61].

The above results will be used to set theoretical priors for the MCMC analysis.

\section{COSMOLOGICAL PERTURBATIONS}

In this section, we discuss the evolution of scalar cosmological perturbations in the $\mathrm{BH}$ model for the perturbed line element given by Eq. (3.2). We introduce the two gauge-invariant gravitational potentials:

$$
\Psi \equiv \delta N+\dot{\psi}, \quad \Phi \equiv-\zeta-H \psi .
$$

For the matter sector, we consider scalar perturbations of the matter-energy momentum tensor $T_{\nu}^{\mu}$ arising from the action $\mathcal{S}_{M}$, as $\delta T_{0}^{0}=-\delta \rho, \delta T_{i}^{0}=\partial_{i} \delta q$, and $\delta T_{j}^{i}=\delta P \delta_{j}^{i}$. The density perturbation $\delta \rho$, the momentum perturbation $\delta q$, and the pressure perturbation $\delta P$ are expressed in terms 
of the sum of each matter component, as $\delta \rho=\sum_{i} \delta \rho_{i}$, $\delta q=\sum_{i} \delta q_{i}$, and $\delta P=\sum_{i} \delta P_{i}$, where $i=m, r$. We introduce the gauge-invariant density contrast:

$$
\Delta_{i} \equiv \frac{\delta \rho_{i}}{\rho_{i}}-3 H \frac{\delta q_{i}}{\rho_{i}},
$$

where $\rho_{i}$ is the background density of each component. In the $\mathrm{BH}$ model, the full linear perturbation equations of motion were derived in Ref. [61].

In Fourier space with the comoving wave number $k$, we relate the gravitational potentials in Eq. (4.1) with the total matter density contrast $\Delta=\sum_{i} \Delta_{i}$, as [90-92]

$$
\begin{gathered}
-k^{2} \Psi=4 \pi G_{\mathrm{N}} a^{2} \mu(a, k) \rho \Delta, \\
-k^{2}(\Psi+\Phi)=8 \pi G_{\mathrm{N}} a^{2} \Sigma(a, k) \rho \Delta,
\end{gathered}
$$

where $G_{\mathrm{N}}$ is the Newton gravitational constant given by Eq. (3.32), and $\rho=\sum_{i} \rho_{i}$ is the total background matter density. The dimensionless quantities $\mu$ and $\Sigma$ correspond to the effective gravitational couplings felt by matter and light, respectively. For nonrelativistic matter, the density contrast $\Delta_{m}$ obeys [61]

$$
\ddot{\Delta}_{m}+2 H \dot{\Delta}_{m}+\frac{k^{2}}{a^{2}} \Psi=-3(\ddot{\mathcal{B}}+2 H \dot{\mathcal{B}}),
$$

where $\mathcal{B} \equiv \zeta+H \delta q_{m} / \rho_{m}$. This means that the matter density contrast grows due to the gravitational instability through the modified Poisson equation (4.3). In GR, both $\mu$ and $\Sigma$ are equivalent to 1 , but in the BH model, they are different from 1 . Hence the growth of structures and gravitational potentials is subject to modifications.

For the perturbations deep inside the sound horizon $\left(c_{s}^{2} k^{2} / a^{2} \gg H^{2}\right)$, the common procedure is to resort to a quasistatic approximation for the estimations of $\mu$ and $\Sigma$ [93-95]. This amounts to picking up the terms containing $k^{2} / a^{2}$ and $\Delta_{m}$ in the perturbation equations of motion. In Horndeski theories, it is possible to obtain the closed form expressions of $\Psi, \Phi, \zeta[19,95]$. In GLPV theories, the additional time derivatives $\alpha_{\mathrm{H}} \dot{\psi}$ and $\alpha_{\mathrm{H}} \dot{\zeta}$ appear even under the quasistatic approximation $[31,96]$, so the perturbation equations are not closed. If $\left|\alpha_{\mathrm{H}}\right|$ is very much smaller than 1 and $x_{4}$ is subdominant to $x_{1,2,3}$, we may ignore the contributions of the term $x_{4}$ to the perturbation equations. In this case, we can estimate $\mu$ and $\Sigma$ in the BH model, as [61]

$$
\mu \simeq \Sigma \simeq 1+\frac{2 Q_{t} x_{3}^{2}}{Q_{s} c_{s}^{2}\left(2-x_{3}\right)^{2}} .
$$

Since $\mu$ and $\Sigma$ are identical to each other, it follows that $\Psi \simeq \Phi$. Under the theoretically consistent conditions (3.29), (3.30), and (3.33), we also have $\mu \simeq \Sigma>1$ and hence the gravitational interaction is stronger than that in GR. Let us note that in the following we will not rely on this approximation and we will solve the complete linear perturbation equations.

To understand the evolution of perturbations, we consider four different cases (BH1, BH2, BH3, GGC) listed in Table I. The difference between these models is characterized by the different choices of initial conditions $x_{i}^{(s)}$ at the redshift $z_{s}=1.5 \times 10^{5}$. Among them, BH1 has the largest initial value $x_{4}^{(s)}$, while $x_{4}$ is always 0 in GGC (which belongs to Horndeski theories). In Fig. 2, we plot the evolution of $x_{i}$ from the past to today for these four different cases. In BH1, the variable $x_{4}$ dominates over other variables $x_{1,2,3}$ for $a \lesssim 10^{-2}$, but it becomes subdominant at low redshifts with today's value of order $10^{-5}$. Comparing BH1 with $\mathrm{BH} 3$, we observe that the initial largeness of $x_{4}$ does not necessarily imply the large present-day value $x_{4}^{(0)}$. At low redshifts, $x_{4}$ is typically less than the order $10^{-3}$ to avoid $c_{s}^{2}<0$ with the amplitude smaller than $x_{1,2,3}$, in which case the analytic estimation (4.6) can be trustable. Indeed, for all the models given in Table I, we numerically checked that the quasistatic approximation holds with subpercent precision for the wave numbers $k>0.01 \mathrm{Mpc}^{-1}$ (as confirmed in Horndeski theories in Refs. $[89,97])$.

In the top panel of Fig. 3, we plot the evolution of $\Psi$ normalized by its initial value $\Psi^{(s)}$ for the four models in Table I and for the $\Lambda$ CDM. In the bottom panel, we depict the percentage difference of $\Psi$ for the chosen models with respect to $\Lambda \mathrm{CDM}$. At the late epoch, the deviations from $\Lambda \mathrm{CDM}$ show up with the enhanced gravitational potential (around $a \sim 0.2$ for the $\mathrm{BH} 2, \mathrm{BH} 3, \mathrm{GGC}$ models). The largest deviation arises for $\mathrm{BH} 3$, in which case the difference is more than $75 \%$ today. As estimated from Eq. (4.6),

TABLE I. List of starting values of the density parameters $x_{i}$ at the redshift $z_{s}=1.5 \times 10^{5}$ and corresponding today's values for three $\mathrm{BH}$ models and the GGC model with $x_{4}=0$. The BH1, $\mathrm{BH} 2$ and $\mathrm{BH} 3$ models differ in the starting values $x_{i}^{(s)}$. All of them satisfy theoretically consistent conditions discussed in Sec. IV. We study these models for the purpose of visualizing and quantifying the modifications from $\Lambda \mathrm{CDM}$. The cosmological parameters (e.g., $H_{0}, \Omega_{m}, \Omega_{r}$ ) used for these models are the Planck 2015 best-fit values for $\Lambda$ CDM [98].

\begin{tabular}{lcccc}
\hline \hline Parameters & BH1 & BH2 & BH3 & GGC \\
\hline$x_{1}^{(s)}\left(\times 10^{-16}\right)$ & -1 & -0.1 & -0.01 & -1 \\
$x_{2}^{(s)}\left(\times 10^{-16}\right)$ & 5 & 0.05 & 0.0001 & 5 \\
$x_{3}^{(s)}\left(\times 10^{-9}\right)$ & 1 & 1 & 0.1 & 10 \\
$x_{4}^{(s)}\left(\times 10^{-6}\right)$ & 100 & 1 & 1 & 0 \\
$x_{1}^{(0)}$ & -1.37 & -1.03 & -0.73 & -1.23 \\
$x_{2}^{(0)}$ & 2.03 & 1.02 & 0.12 & 1.63 \\
$x_{3}^{(0)}$ & 0.03 & 0.69 & 1.30 & 0.29 \\
$x_{4}^{(0)}$ & $1 \times 10^{-5}$ & $5 \times 10^{-6}$ & $2 \times 10^{-4}$ & 0 \\
\hline \hline
\end{tabular}




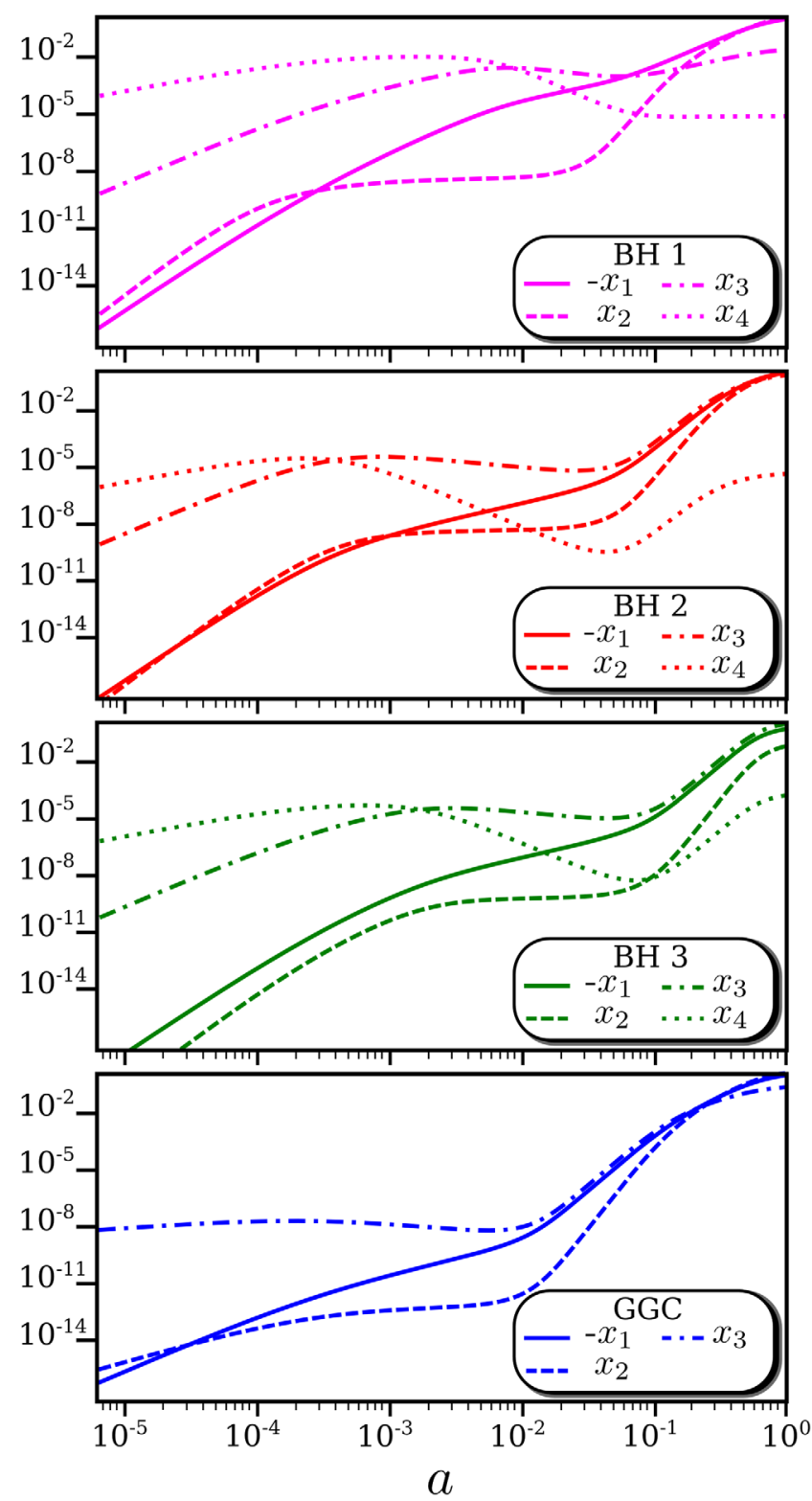

FIG. 2. Evolution of the dimensionless variables defined in Eq. (3.15) versus the scale factor $a$ (with today's value 1 ) for four test models listed in Table I. In this table, the staring values of parameters $x_{i}$ at the initial redshift $z_{s}=1.5 \times 10^{5}$ are shown for each test model. We discuss physical implications for the evolutions of $x_{i}$ in Sec. IV.

the modified evolution of $\Psi$ is mostly attributed to the cubic Galileon term $x_{3}$. For larger today's values of $x_{3}^{(0)}$, the difference of $\Psi$ from $\Lambda \mathrm{CDM}$ tends to be more significant with the larger deviation of $\mu$ from 1. In Fig. 3, we observe that the deviation from $\Lambda \mathrm{CDM}$ increases with the order of $\mathrm{BH} 1, \mathrm{GGC}, \mathrm{BH} 2, \mathrm{BH} 3$, by reflecting their increasing values of $x_{3}^{(0)}$ given in Table I.

In BH1, there is the suppression of $|\Psi|$ in comparison to $\Lambda \mathrm{CDM}$ at high redshifts $\left(a \lesssim 10^{-2}\right)$. This property arises from the dominance of $x_{4}$ over $x_{1,2,3}$ at early times, in which
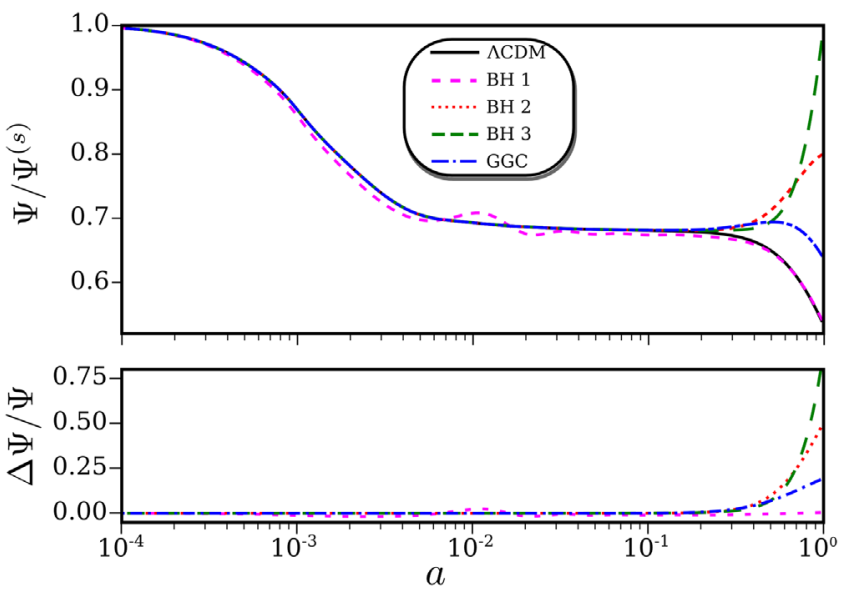

FIG. 3. (Top) Evolution of the gravitational potential $\Psi$ normalized by its initial value $\Psi^{(s)}$ for the wave number $k=0.01 \mathrm{Mpc}^{-1}$. We show the evolution of $\Psi / \Psi^{(s)}$ for four models listed in Table I and also for $\Lambda$ CDM (black line). (Bottom) Percentage relative difference of $\Psi$ relative to that in $\Lambda \mathrm{CDM}$. The cosmological parameters used for this plot are the Planck 2015 best-fit values for $\Lambda$ CDM [98] (which is also the case for plots in Figs. 5 and 6). The physical interpretation of this figure is discussed in Sec. IV.

case the relative density abundances between dark energy and matter fluids are modified. Besides this effect, the non-negligible early-time contribution of $x_{4}$ to scalar perturbations gives rise to a scale-dependent evolution of gravitational potentials, which manifests itself in the $k$-dependent variation of $\mu(a, k)$ and $\Sigma(a, k)$. In Fig. 4, we plot the evolution of $\Psi$ in $\mathrm{BH} 1$ for three different values of $k$. For perturbations on smaller scales, the deviation from

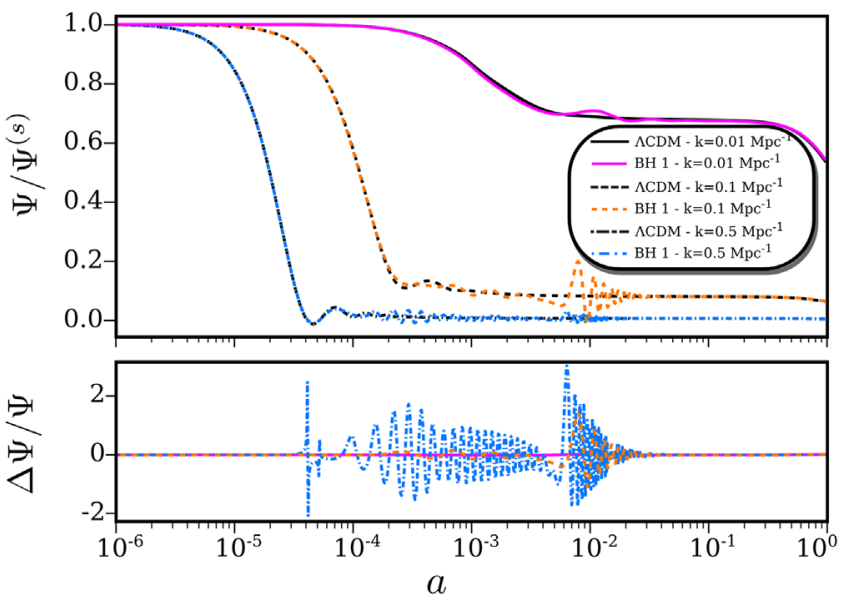

FIG. 4. (Top) Evolution of the gravitational potential $\Psi$ normalized by its initial value $\Psi^{(s)}$ for $\mathrm{BH} 1$ and $\Lambda$ CDM with three different wave numbers: $k=0.01,0.1,0.5 \mathrm{Mpc}^{-1}$. In Table I, we list the starting values of parameters $x_{i}$ at the initial redshift $z_{s}=1.5 \times 10^{5}$ for the $\mathrm{BH} 1$ model. (Bottom) Percentage relative difference of $\Psi$ relative to that in $\Lambda \mathrm{CDM}$ for the same values of $k$ in the top panel. 
$\Lambda \mathrm{CDM}$ tends to be more significant. In models $\mathrm{BH} 2, \mathrm{BH} 3$, and GGC, the early-time evolution of $\Psi$ is similar to that in $\Lambda \mathrm{CDM}$, but they exhibit large deviations from $\Lambda \mathrm{CDM}$ at late times.

At low redshifts, the lensing gravitational potential $\phi_{\text {len }}=(\Psi+\Phi) / 2$ evolves in a similar way to $\Psi$, by reflecting the property $\mu \simeq \Sigma$ for $x_{4}^{(0)} \ll 1$. The lensing angular power spectrum can be computed by using the line of sight integration method, with the convention [99]

$$
C_{\ell}^{\phi \phi}=4 \pi \int \frac{\mathrm{d} k}{k} \mathcal{P}(k)\left[\int_{0}^{\chi_{*}} \mathrm{~d} \chi S_{\phi}\left(k ; \tau_{0}-\chi\right) j_{\ell}(k \chi)\right]^{2},
$$

where $\mathcal{P}(k)=\Delta_{\mathcal{R}}^{2}(k)$ is the primordial power spectrum of curvature perturbations, and $j_{\ell}$ is the spherical Bessel function. The source $S_{\phi}$ is expressed in terms of the transfer function

$$
S_{\phi}\left(k ; \tau_{0}-\chi\right)=2 T_{\phi}\left(k ; \tau_{0}-\chi\right)\left(\frac{\chi_{*}-\chi}{\chi_{*} \chi}\right),
$$

with $T_{\phi}(k, \tau)=k \phi_{\text {len }}, \chi$ is the comoving distance with $\chi_{*}$ corresponding to that to the last scattering surface, $\tau_{0}$ is today's conformal time $\tau=\int a^{-1} \mathrm{~d} t$ satisfying the relation $\chi=\tau_{0}-\tau$. In Fig. 5, we show the lensing power spectra $D_{\ell}^{\phi \phi}=\ell(\ell+1) C_{\ell}^{\phi \phi} /(2 \pi)$ and relative differences in units of the cosmic variance for four models listed in Table I. Since $\Sigma>1$ at low redshifts in BH and GGC models, this works to enhance $D_{\ell}^{\phi \phi}$ compared to $\Lambda \mathrm{CDM}$. We note that the amplitude of matter density contrast $\delta_{m}$ in these models also gets larger than that in $\Lambda$ CDM by reflecting the fact that $\mu>1$. In Fig. 5, we observe that, apart from $\mathrm{BH} 1$ in

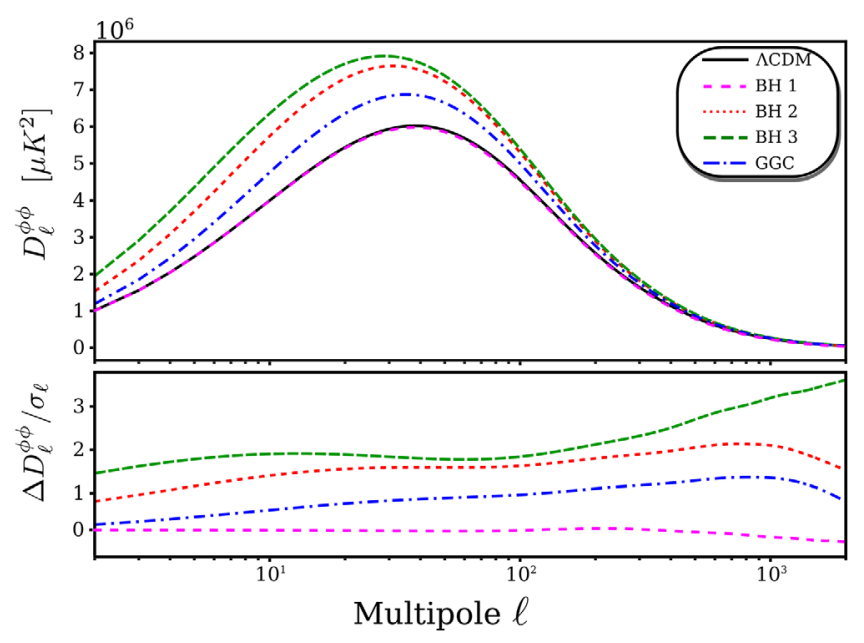

FIG. 5. (Top) Lensing angular power spectra $D_{\ell}^{\phi \phi}=\ell(\ell+$ 1) $C_{\ell}^{\phi \phi} /(2 \pi)$ for $\Lambda \mathrm{CDM}$ and the models listed in Table I, where $C_{\ell}$ is defined by Eq. (4.7). (Bottom) Relative difference of the lensing angular power spectra, computed with respect to $\Lambda \mathrm{CDM}$, in units of the cosmic variance $\sigma_{\ell}=\sqrt{2 /(2 \ell+1)} C_{\ell}^{\Lambda \mathrm{CDM}}$. which $\Sigma$ is close to 1 , the lensing power spectra in other three cases are subject to the enhancement with respect to $\Lambda \mathrm{CDM}$. Since today's values of $\mu$ and $\Sigma$ increase for larger $x_{3}^{(0)}$, the deviation from $\Lambda \mathrm{CDM}$ tends to be more significant with the order of GGC, $\mathrm{BH} 2$, and $\mathrm{BH} 3$.

Let us proceed to the discussion of the impact of $\mathrm{BH}$ and GGC models on the CMB temperature anisotropies. The CMB temperature-temperature (TT) angular spectrum can be expressed as [100]

$$
C_{\ell}^{\mathrm{TT}}=(4 \pi)^{2} \int \frac{\mathrm{d} k}{k} \mathcal{P}(k)\left|\Delta_{\ell}^{\mathrm{T}}(k)\right|^{2},
$$

where

$$
\Delta_{\ell}^{\mathrm{T}}(k)=\int_{0}^{\tau_{0}} \mathrm{~d} \tau e^{i k \tilde{\mu}\left(\tau-\tau_{0}\right)} S_{\mathrm{T}}(k, \tau) j_{\ell}\left[k\left(\tau_{0}-\tau\right)\right],
$$

with $\tilde{\mu}$ being the angular separation, and $S_{\mathrm{T}}(k, \tau)$ is the radiation transfer function. The contribution to $S_{\mathrm{T}}(k, \tau)$ arising from the integrated-Sachs-Wolfe (ISW) effect is of the form

$$
S_{\mathrm{T}}(k, \tau) \sim\left(\frac{\mathrm{d} \Psi}{\mathrm{d} \tau}+\frac{\mathrm{d} \Phi}{\mathrm{d} \tau}\right) e^{-\kappa},
$$

where $\kappa$ is the optical depth. Besides the early ISW effect which occurs during the transition from the radiation to matter eras by the time variation of $\Psi+\Phi$, the presence of dark energy induces the late-time ISW effect. In the $\Lambda$ CDM model, the gravitational potential $-(\Psi+\Phi)$, which is positive, decreases by today with at least more than $30 \%$ relative to its initial value (see Fig. 3). As we observe in Fig. 6 we have $\dot{\Psi}+\dot{\Phi}>0$ in this case, so the ISW effect

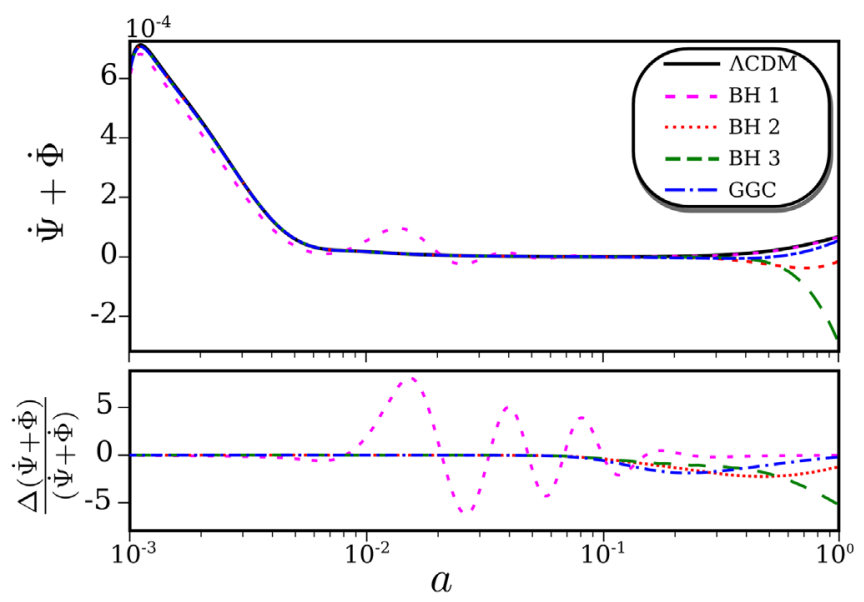

FIG. 6. (Top) Evolution of the time derivative $\dot{\Psi}+\dot{\Phi}$ for $\Lambda \mathrm{CDM}$ and the models listed in Table I, computed at $k=0.01 \mathrm{Mpc}^{-1}$. (Bottom) Relative difference of $\dot{\Psi}+\dot{\Phi}$, computed with respect to $\Lambda \mathrm{CDM}$. See the discussion after Eq. (4.11) for the physical interpretation of this figure. 


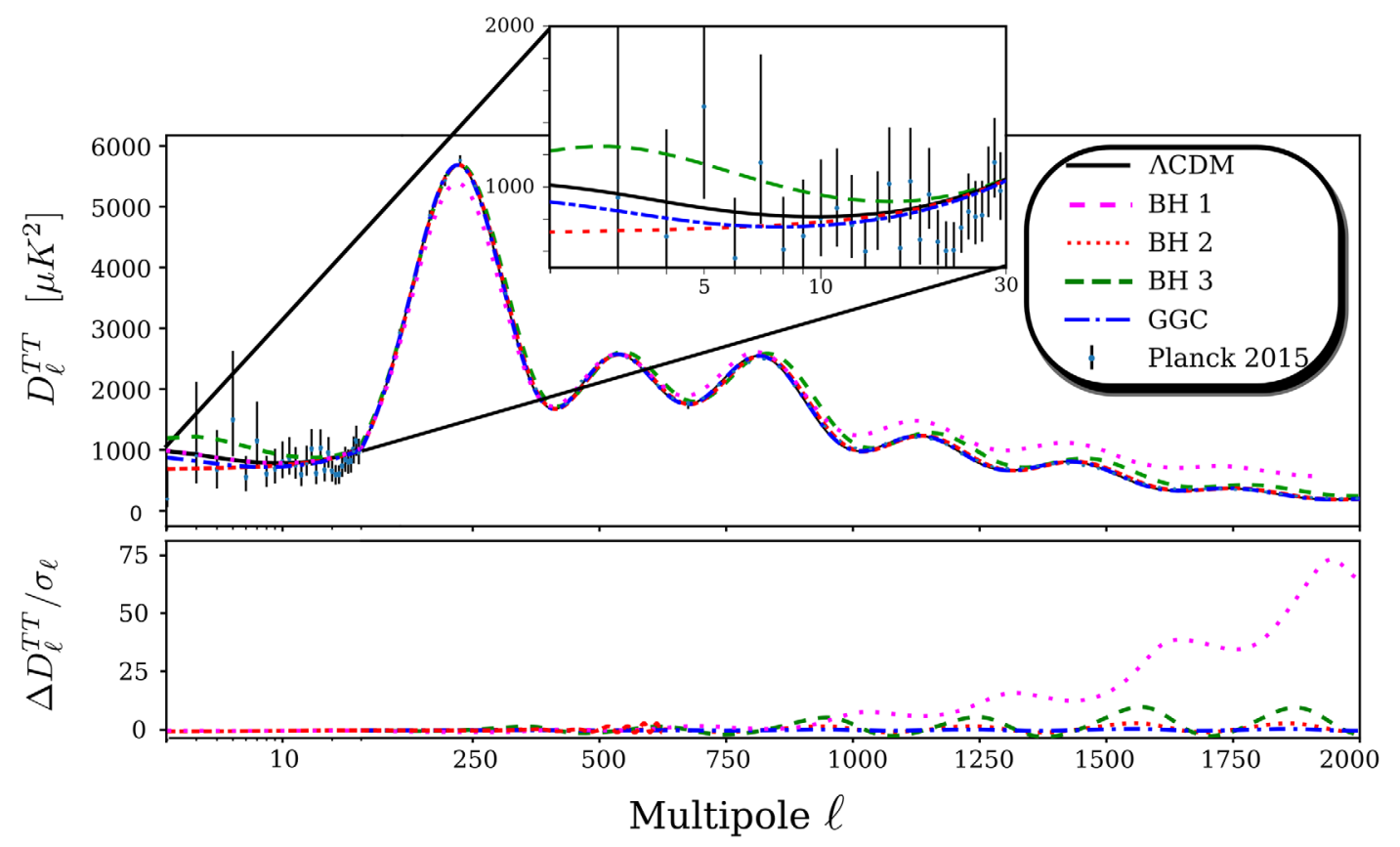

FIG. 7. (Top) CMB TT power spectra $D_{\ell}^{\mathrm{TT}}=\ell(\ell+1) C_{\ell}^{\mathrm{TT}} /(2 \pi)$ for the test models presented in Table I, compared with data points from the Planck 2015 release. (Bottom) Relative difference of TT power spectra, computed with respect to $\Lambda$ CDM in units of the cosmic variance $\sigma_{\ell}=\sqrt{2 /(2 \ell+1)} C_{\ell}^{\Lambda \mathrm{CDM}}$.

gives rise to the positive contribution to Eq. (4.9). In Fig. 7, we plot the $\mathrm{CMB}$ TT power spectra $D_{\ell}^{\mathrm{TT}}=\ell(\ell+$ 1) $C_{\ell}^{\mathrm{TT}} /(2 \pi)$ for the models listed in Table I and $\Lambda \mathrm{CDM}$. In BH1 the parameter $\Sigma$ is close to 1 at low redshifts due to the smallness of $x_{3}^{(0)}$, so the late-time ISW effect works in the similar way to the GR case. Hence the TT power spectrum in $\mathrm{BH} 1$ for the multipoles $\ell \lesssim 30$ is similar to that in $\Lambda \mathrm{CDM}$.

In the GGC model of Fig. 7, we observe that the largescale ISW tail is suppressed relative to that in $\Lambda \mathrm{CDM}$. This reflects the fact that the larger deviation of $\Sigma$ from 1 leads to the time derivative $\dot{\Psi}+\dot{\Phi}$ closer to 0, see Fig. 6. Hence the late-time ISW effect is not significant, which results in the suppression of $D_{\ell}^{\mathrm{TT}}$ with respect to $\Lambda \mathrm{CDM}$. In Ref. [71] this fact was first recognized in the GGC model, which exhibits a better fit to the Planck CMB data. As the deviation of $\Sigma$ from 1 increases further, the sign of $\dot{\Psi}+\dot{\Phi}$ changes to be negative (see Fig. 6). The BH2 model can be regarded as such a marginal case in which the large-scale ISW tail is nearly flat. In BH3, the increase of $\Sigma$ at low redshifts is so significant that the largely negative ISW contribution to Eq. (4.9) leads to the enhanced low$\ell$ TT power spectrum relative to $\Lambda$ CDM.

The modified evolution of the Hubble expansion rate from $\Lambda \mathrm{CDM}$ generally leads to the shift of CMB acoustic peaks at high $\ell$. In Fig. 8, we observe that the largest deviation of $H(a)$ at high redshifts occurs for BH1 by the dominance of $x_{4}$ over $x_{1,2,3}$. This leads to the shift of acoustic peaks toward lower multipoles (see Fig. 7).
We also find that $\mathrm{BH} 3$ is subject to non-negligible shifts of high- $\ell$ peaks due to the large modification of $H(a)$ at low redshifts, in which case the peaks shift toward higher multipoles. Moreover, there is the large enhancement of ISW tails for BH3, so it should be tightly constrained from the CMB data. We note that the shift of CMB acoustic peaks is further constrained by the datasets of $\mathrm{BAO}$ and $\mathrm{SN}$ Ia. For $\mathrm{BH} 2$ and GGC the changes of peak positions are small in comparion to $\mathrm{BH} 1$ and $\mathrm{BH} 3$, but still they are in

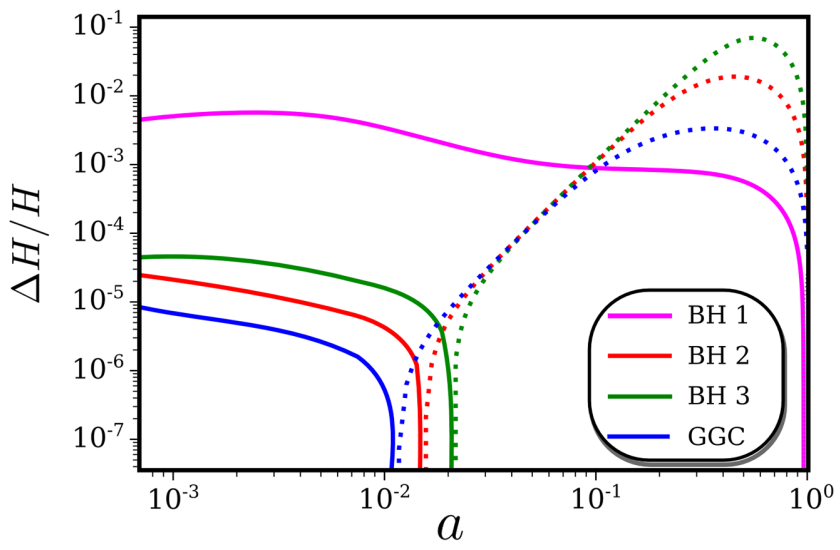

FIG. 8. Evolution of the relative Hubble rate for the models listed in Table I compared to $\Lambda$ CDM. The solid lines correspond to a positive difference, whereas the opposite holds for the dashed lines. For BH1 the largest difference from $\triangle \mathrm{CDM}$ occurs in the early cosmological epoch, in which case the CMB acoustic peaks shift toward lower multipoles. 
the range testable by the CMB data. Moreover, the largescale ISW tail is subject to the suppression relative to $\Lambda \mathrm{CDM}$ in $\mathrm{BH} 2$ and GGC.

In BH1, we also notice a change in the amplitude of acoustic peaks occurring dominantly at high $\ell$. This is known to be present in models with early-time modifications of gravity [101,102]. The modification of gravitational potentials affects the evolution of radiation perturbations (monopole and dipole) through the radiation driving effect [101,103], thus resulting in the changes in amplitude and phase of acoustic peaks at high $\ell$.

The modified time variations of $\Psi$ and $\Phi$ around the recombination epoch also give a contribution to the early ISW effect. This is important on scales around the first acoustic peak, corresponding to the wave number $k \simeq 0.016 \mathrm{Mpc}^{-1}$ for our choice of model parameters. To have a more qualitative feeling of this contribution, we have estimated the impact of the early ISW effect on $D_{\ell}^{\mathrm{TT}}$ by using the approximate ISW integral presented in Ref. [103]:

$$
\left.\int_{\tau_{*}}^{\tau_{0}} \mathrm{~d} \tau\left(\frac{\mathrm{d} \Psi}{\mathrm{d} \tau}+\frac{\mathrm{d} \Phi}{\mathrm{d} \tau}\right) j_{\ell}\left[k\left(\tau_{0}-\tau\right)\right] \simeq[\Psi+\Phi]\right|_{\tau_{*}} ^{\tau_{0}} j_{\ell}\left(k \tau_{0}\right),
$$

where $\tau_{*}$ is the conformal time at the last scattering. Then, we find a negative difference of about $4.9 \%$ between $\mathrm{BH} 1$ and $\Lambda \mathrm{CDM}$. This is in perfect agreement with the change in amplitude of the first acoustic peak shown in Fig. 7. Thus, the $\mathrm{BH}$ models in which $x_{4}$ is the dominant contribution to the dark energy dynamics at early times can be severely constrained from the CMB data.

We stress that, in the late Universe, $x_{4}$ is typically suppressed compared to $x_{1,2,3}$ for the viable cosmological background, so the main impact on the evolution of perturbations comes from the cubic Galileon term $x_{3}$. The analytic estimation (4.6) is sufficiently trustable for studying the evolution of gravitational potentials and matter perturbations at low redshifts. However, we solve the full perturbation equations of motion for the MCMC analysis without resorting to the quasistatic approximation.

\section{OBSERVATIONAL CONSTRAINTS}

We place observational bounds on the $\mathrm{BH}$ model by performing the MCMC simulation with different combinations of datasets at high and low redshifts.

\section{A. Datasets}

For the MCMC likelihood analysis, based on the EFTCosmoMC code, we use the Planck 2015 [98,104] data of CMB temperature and polarization on large angular scales, for multipoles $\ell<29$ (low- $\ell$ TEB likelihood) and the CMB temperature on smaller angular scales (PLIK TT Likelihood). We also consider the BAO measurements from the 6dF Galaxy Survey [105] and from the SDSS DR7 main galaxy sample [106]. Moreover, we include the combined BAO and RSD datasets from the SDSS DR12 consensus release [107] and the JLA SN Ia sample [108]. We will refer to the full combined datasets as "PBRS."

Finally, we impose the flat priors on the model parameters: $x_{1}^{(s)} \in[-10,10] \times 10^{-16}, \quad x_{3}^{(s)} \in[-10,10] \times 10^{-9}$, and $x_{4}^{(s)} \in[0,10] \times 10^{-6}$. Even by increasing the prior volume by 1 order of magnitude, we confirmed that the likelihood results are not subject to the priors choice.

\section{B. Constrained parameter space}

In this section, we show observational constraints on model parameters in the $\mathrm{BH}$ model. We use the datasets presented in Sec. VA with two combinations: (i) Planck and (ii) PBRS. For reference, we also present the results of the $\Lambda$ CDM model.

In Table II, we show the marginalized values of today's four density parameters $x_{i}^{(0)}$ with $95 \%$ confidence level (CL) limits. In Fig. 9, we plot the observationally allowed regions derived by two combinations of datasets with the $68 \%$ and $95 \%$ CL boundaries. The best-fit values of $x_{1}^{(0)}$ and $x_{2}^{(0)}$ constrained by the Planck data are not affected much by including the datasets of BAO, SN Ia, and RSDs. In the observationally allowed region we have $x_{1}^{(0)}<0$ and $x_{2}^{(0)}>0$, but there are neither ghosts nor Laplacian instabilities in the constrained parameter space (as in the ghost condensate model [76]).

With the Planck data alone, the 95\% CL upper bound on $x_{3}^{(0)}$ is close to 1 , but the PBRS datasets give the tighter limit $x_{3}^{(0)} \leq 0.27$ at $95 \%$ CL. The maximum likelihood value of $x_{3}^{(0)}$ derived with the Planck data is 0.34 , which is similar to the corresponding value 0.27 constrained with PBRS. The nonvanishing best-fit value of $x_{3}^{(0)}$ is attributed to the facts that, relative to $\Lambda \mathrm{CDM}$, (i) the Galileon term can suppress the large-scale ISW tale, and (ii) the modified background evolution gives rise to the TT power spectrum showing a better fit to the Planck CMB data at high $\ell$. In Fig. 10, these properties can be seen in the best-fit TT power spectrum of the $\mathrm{BH}$ model. Increasing $x_{3}^{(0)}$ further eventually leads to

TABLE II. Marginalized values of the model parameters $x_{i}^{(0)}$ and their 95\% CL bounds, derived by Planck and PBRS datasets. In parenthesis, we also show the maximum likelihood values of these parameters.

\begin{tabular}{lcc}
\hline \hline Parameters & Planck & PBRS \\
\hline$x_{1}^{(0)}$ & $-1.32_{-0.12}^{+0.21}(-1.25)$ & $-1.35_{-0.06}^{+0.01}(-1.25)$ \\
$x_{2}^{(0)}$ & $1.85_{-0.69}^{+0.33}(1.62)$ & $1.98_{-0.29}^{+0.14}(1.68)$ \\
$x_{3}^{(0)}$ & $0.16_{-0.18}^{+0.54}(0.34)$ & $0.07_{-0.1}^{+0.2}(0.27)$ \\
$x_{4}^{(0)}\left(\times 10^{-6}\right)$ & $0.7_{-1.8}^{+2.2}(0.15)$ & $0.3_{-0.6}^{+0.7}(0.54)$ \\
\hline \hline
\end{tabular}



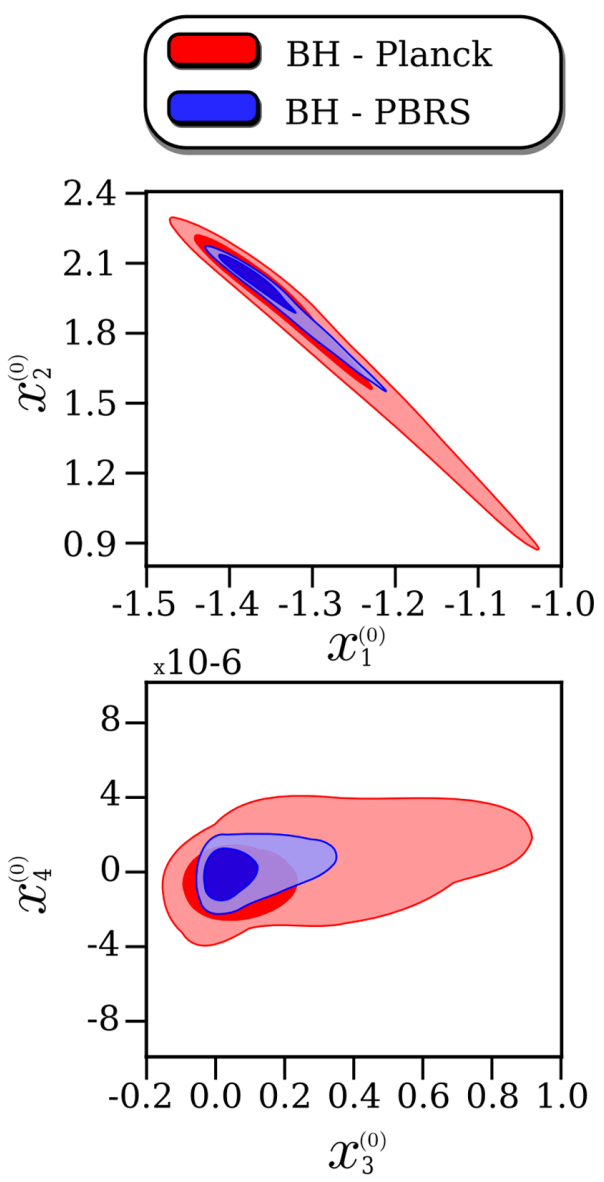

FIG. 9. Two-dimensional observational bounds on the combinations of today's density parameters $\left(x_{1}^{(0)}, x_{2}^{(0)}\right)$ and $\left(x_{3}^{(0)}, x_{4}^{(0)}\right)$. The colored regions correspond to the parameter space constrained by the Planck (red) and PBRS (blue) datasets at $68 \%$ (inside) and 95\% (outside) CL limits.

the enhancement of the ISW tale in comparison to $\Lambda \mathrm{CDM}$. As we see in BH3 of Fig. 7, the models with large $x_{3}^{(0)}$ do not fit the TT power spectrum well at high $\ell$ either. Such models are disfavored from the CMB data (as in the case of covariant Galileons $[109,110])$, so that $x_{3}^{(0)}$ is bounded from above. The RSD data at low redshifts can be also consistent with the intermediate values of $x_{3}^{(0)}$ constrained from CMB.

In Fig. 11, we show the evolution of $w_{\mathrm{DE}}$ for the best-fit BH model. As discussed in Ref. [61], the existence of $x_{2}$ besides $x_{3}$ prevents the approach to a tracker solution characterized by $w_{\mathrm{DE}}=-2$ during the matter-dominated epoch. The best-fit background solution first enters the region $-2<w_{\mathrm{DE}}<-1$ in the matter era and finally approaches a de Sitter attractor characterized by $w_{\mathrm{DE}}=-1$. Thus, the $\mathrm{BH}$ and GGC models with $x_{2} \neq 0$ alleviate the observational incompatibility problem of tracker solutions of covariant Galileons [78]. For the best-fit $\mathrm{BH}$ model, there is the deviation of $w_{\mathrm{DE}}$ from -1 with the value $w_{\mathrm{DE}} \approx-1.1$ at the redshift $1<z<3$, so the model is different from $\Lambda \mathrm{CDM}$ even at the background level.

From the PBRS datasets, today's value of $x_{4}$ is constrained to be

$$
x_{4}^{(0)}=0.3_{-0.6}^{+0.7} \times 10^{-6} \quad(95 \% \mathrm{CL}),
$$

so that $\left|x_{4}^{(0)}\right|$ is at most of order $10^{-6}$. With the Planck data alone, the upper bound of $\left|x_{4}^{(0)}\right|$ is also of the same order. This means that the upper limit of $x_{4}^{(0)}$ is mostly determined by the CMB data. As we discussed in Sec. IV, the CMB TT power spectrum is sensitive to the dominance of $x_{4}$ over $x_{1,2,3}$ in the early cosmological epoch. Then, today's value of $x_{4}$ is also tightly constrained as Eq. (5.1), which translates to the bound

$$
\left|\alpha_{\mathrm{H}}^{(0)}\right| \leq \mathcal{O}\left(10^{-6}\right)
$$

Apart from the constraint arising from the GW decay to dark energy [62], the above upper limit on $\alpha_{\mathrm{H}}^{(0)}$ is the most stringent bound derived from cosmological observations so far.

In Table III, we present the values of $H_{0}, \sigma_{8}^{(0)}$, and $\Omega_{m}^{(0)}$ constrained from the datasets of Planck and PBRS for the $\mathrm{BH}$ and $\Lambda \mathrm{CDM}$ models. The bounds on $H_{0}, \sigma_{8}^{(0)}$, and $\Omega_{m}^{(0)}$ derived with the PBRS datasets are similar to those in $\Lambda \mathrm{CDM}$. In Fig. 12, we also plot the two-dimensional observational contours for these parameters constrained by the Planck data. The direct measurements of $H_{0}$ at low redshifts [111] give the bound $H_{0}>70 \mathrm{~km} \mathrm{sec}^{-1} \mathrm{Mpc}^{-1}$, whereas the Planck data tend to favor lower values of $H_{0}$. Thus, as in the case of $\Lambda \mathrm{CDM}$, the $\mathrm{BH}$ model does not alleviate the tension of $H_{0}$ between the Planck data and its local measurements. The similar property also holds for $\sigma_{8}^{(0)}$, where the Planck data favor higher values of $\sigma_{8}^{(0)}$ than those constrained in low-redshift measurements. We can also put further bounds on $\sigma_{8}^{(0)}$ by using the datasets of weak lensing measurements, such as KiDS [9-11]. For this purpose, we need to take nonlinear effects into account in the MCMC analysis, which is beyond the scope of the current paper.

\section{Model selection}

The $\mathrm{BH}$ model has three more parameters compared to those in $\Lambda \mathrm{CDM}$. This means that the former has more freedom to fit the model better with the data. In order to study whether the former is statistically favored over the latter, we compute the deviance information criterion (DIC) [112]:

$$
\mathrm{DIC}=\chi_{\mathrm{eff}}^{2}(\hat{\theta})+2 p_{\mathrm{D}}
$$




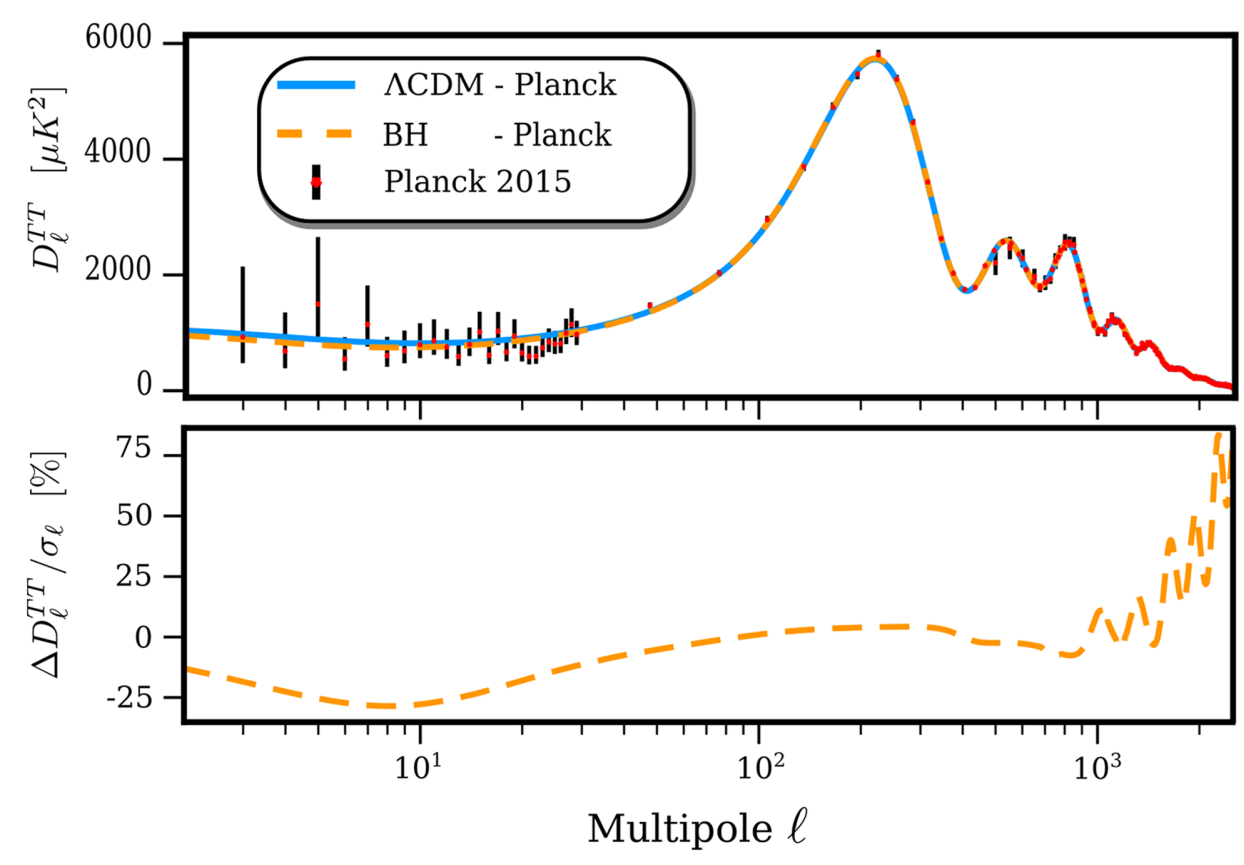

FIG. 10. (Top) Best-fit CMB TT power spectra $D_{\ell}^{\mathrm{TT}}=\ell(\ell+1) C_{\ell}^{\mathrm{TT}} /(2 \pi)$ for $\mathrm{BH}$ and $\Lambda \mathrm{CDM}$, obtained with the Planck dataset. The model parameters used for this plot are given in Tables II and III. For comparison, we plot the data points from the Planck 2015 release [98]. (Bottom) Relative difference of the best-fit TT power spectra, in units of the cosmic variance $\sigma_{\ell}=\sqrt{2 /(2 \ell+1)} C_{\ell}^{\Lambda \mathrm{CDM}}$. See Sec. V B for the difference between the best-fit BH and $\Lambda$ CDM models.

where $\chi_{\text {eff }}^{2}(\hat{\theta})=-2 \ln \mathcal{L}(\hat{\theta})$, and $\hat{\theta}$ is a vector associated with model parameters maximizing the likelihood function $\mathcal{L}$. The quantity $p_{\mathrm{D}}$ is defined by $p_{\mathrm{D}}=\bar{\chi}_{\text {eff }}^{2}(\theta)-\chi_{\text {eff }}^{2}(\hat{\theta})$, where the bar represents an average over the posterior distribution. From its definition, the DIC accounts for the goodness of fit, $\chi_{\mathrm{eff}}^{2}(\hat{\theta})$, and the Bayesian complexity of the model, $p_{\mathrm{D}}$. The complex models with more free parameters give larger $p_{\mathrm{D}}$. To compare the $\mathrm{BH}$ model with the $\Lambda \mathrm{CDM}$ model, we calculate

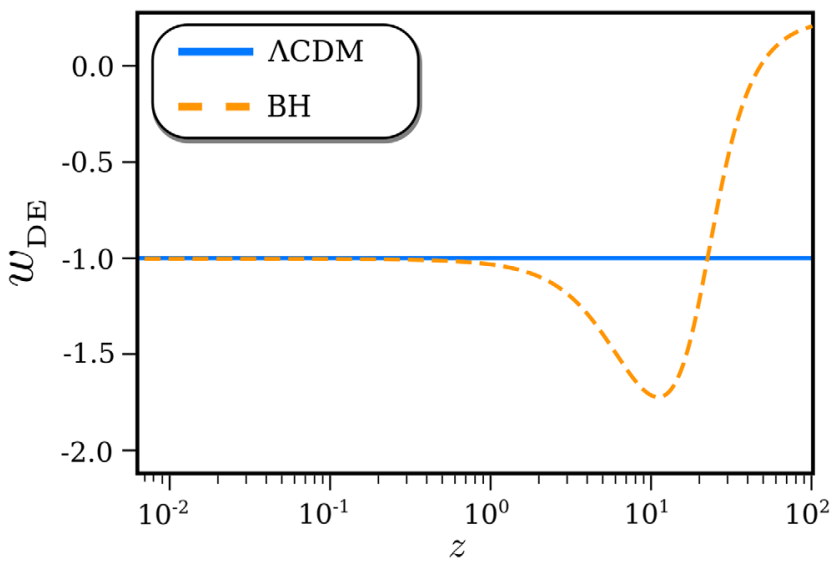

FIG. 11. Best-fit evolution of the dark energy equation of state $w_{\mathrm{DE}}$ for $\mathrm{BH}$ and $\Lambda \mathrm{CDM}$, obtained from the PBRS analysis. The model parameters used for this plot are given in Tables II and III. In the best-fit $\mathrm{BH}, w_{\mathrm{DE}}$ first enters the region $w_{\mathrm{DE}}<-1$ and then it finally approaches the asymptotic value $w_{\mathrm{DE}}=-1$.

$$
\Delta \mathrm{DIC}=\mathrm{DIC}_{\mathrm{BH}}-\mathrm{DIC}_{\Lambda \mathrm{CDM}} .
$$

If $\triangle \mathrm{DIC}$ is negative, then $\mathrm{BH}$ is favored over $\Lambda \mathrm{CDM}$. For positive $\triangle \mathrm{DIC}$, the situation is reversed.

In Table IV, we present the relative differences of $\Delta \chi_{\text {eff }}^{2}$ and $\triangle \mathrm{DIC}$ in $\mathrm{BH}$ and GGC models, as compared to $\Lambda \mathrm{CDM}$. Since $\Delta \chi_{\text {eff }}^{2}$ are always negative, these models provide the better fit to the data relative to $\Lambda$ CDM. In particular, we find that $\Delta \chi_{\text {eff }}^{2}$ constrained by the Planck data alone are smaller than those derived with the PBRS datasets. This preference of $\mathrm{BH}$ over $\Lambda \mathrm{CDM}$ by the Planck data arises from combined effects of the suppressed large-scale ISW tale caused by the Galileon term and the modified high $\ell$ TT power spectrum induced by the

TABLE III. Marginalized values of $H_{0}, \sigma_{8}^{(0)}$, and $\Omega_{m}^{(0)}$ and their 95\% CL bounds in the $\mathrm{BH}$ and $\Lambda \mathrm{CDM}$ models, derived by Planck and PBRS datasets. The unit of $H_{0}$ is $\mathrm{km} \mathrm{sec}^{-1} \mathrm{Mpc}^{-1}$. In parenthesis, we also show maximum likelihood values of these parameters.

\begin{tabular}{lccc}
\hline \hline Parameter & Model & Planck & PBRS \\
\hline$H_{0}$ & $\mathrm{BH}$ & $68.7_{-2.8}^{+3.2}(69.6)$ & $68.0_{-1.1}^{+1.1}(68.2)$ \\
& $\Lambda \mathrm{CDM}$ & $67.9 \pm 2.0(67.6)$ & $68 \pm 1(68)$ \\
$\sigma_{8}^{(0)}$ & $\mathrm{BH}$ & $0.849_{-0.035}^{+0.037}(0.87)$ & $0.84 \pm 0.03(0.84)$ \\
& $\Lambda \mathrm{CDM}$ & $0.841 \pm 0.03(0.83)$ & $0.84 \pm 0.03(0.84)$ \\
$\Omega_{m}^{(0)}$ & $\mathrm{BH}$ & $0.300_{-0.034}^{+0.033}(0.28)$ & $0.306_{-0.014}^{+0.014}(0.30)$ \\
& $\Lambda \mathrm{CDM}$ & $0.30 \pm 0.03(0.31)$ & $0.31 \pm 0.01(0.31)$ \\
\hline \hline
\end{tabular}




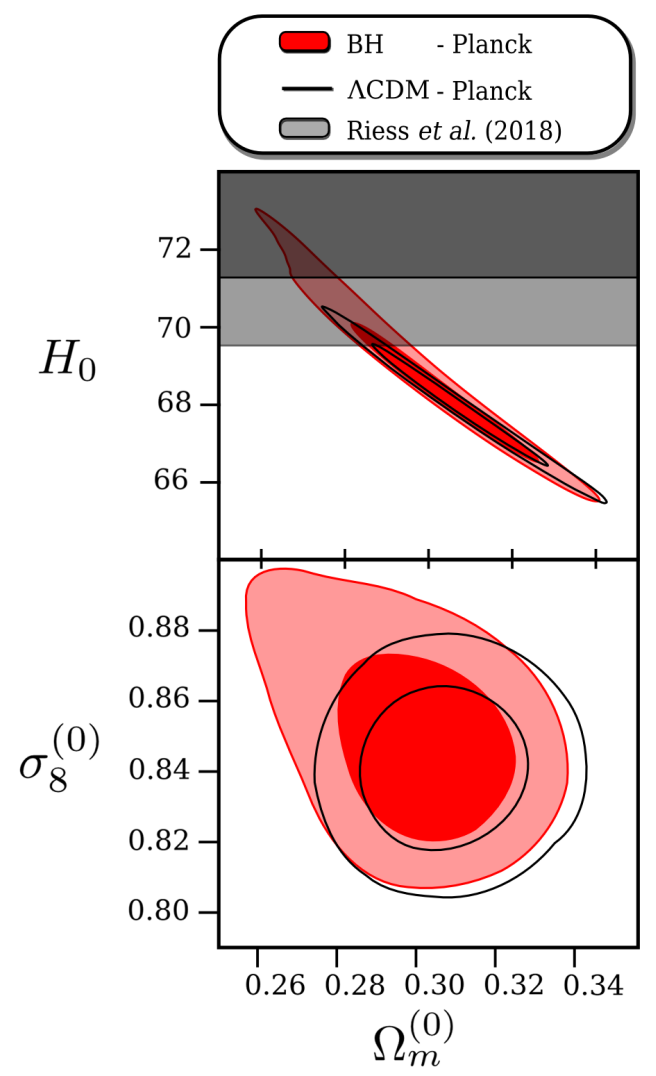

FIG. 12. The $68 \%$ and $95 \%$ CL two-dimensional bounds on $\left(H_{0}, \Omega_{m}^{(0)}\right)$ (top) and $\left(\sigma_{8}^{(0)}, \Omega_{m}^{(0)}\right)$ (bottom) constrained by the Planck 2015 data, with the unit $\mathrm{km} \mathrm{sec}^{-1} \mathrm{Mpc}^{-1}$ for $H_{0}$. The observational bounds on $\mathrm{BH}$ and $\Lambda \mathrm{CDM}$ models are shown as the red and black colors, respectively. In the top panel, the grey bands represent the $68 \%$ and $95 \%$ CL bounds on $H_{0}$ derived by its direct measurement at low redshifts [111]. See the last paragraph of Sec. V B for the discussion of likelihood results.

different background evolution relative to $\Lambda \mathrm{CDM}$ (as shown in Fig. 10). The former contributes by $\sim 20 \%$ to a better $\chi_{\text {eff }}^{2}$, while the latter to the remaining $\sim 80 \%$. We note that a further lowering of the ISW tail is limited by the shift of acoustic peaks at high $\ell$. Such modifications are also subject to further constraints from the datasets of BAO and SN Ia, but the values of $\Delta \chi_{\text {eff }}^{2}$ constrained with the PBRS datasets are still negative in both BH and GGC models.

TABLE IV. Model comparisons in terms of $\Delta \chi_{\text {eff }}^{2}$ and $\Delta$ DIC. As the reference model, we use the value $\chi_{\text {eff }}^{2}$ in $\Lambda$ CDM. The results for GGC are taken from Ref. [71].

\begin{tabular}{lccc}
\hline \hline Model & Dataset & $\Delta \chi_{\text {eff }}^{2}$ & $\Delta$ DIC \\
\hline BH & Planck & -4.7 & 0.25 \\
BH & PBRS & -1.8 & 0.1 \\
GGC & Planck & -4.8 & -2.5 \\
GGC & PBRS & -2.8 & -0.6 \\
\hline \hline
\end{tabular}

According to the DIC, the $\mathrm{BH}$ model is slightly disfavored over $\Lambda$ CDM with the PBRS datasets. The GGC model, which has one parameter less than those in $\mathrm{BH}$, is favored over $\Lambda$ CDM with both Planck and PBRS datasets. This implies that the existence of an additional parameter $x_{4}$ does not contribute to provide better fits to the data. Indeed, today's value of $x_{4}$ is severely constrained as Eq. (5.1) mostly from the CMB data. At the same time, this implies that there are no observational signatures for the deviation $\alpha_{\mathrm{H}}$ from Horndeski theories. It is interesting to note that the GGC model, which belongs to a subclass of Horndeski theories, is statistically favored over $\Lambda \mathrm{CDM}$ even with two additional parameters, but this property does not persist in the $\mathrm{BH}$ model due to the extra beyond-Horndeski term $\alpha_{\mathrm{H}}$ modifying the cosmic expansion and growth histories.

\section{CONCLUSION}

We studied observational constraints on the BH model given by the action (2.1) with the functions (2.7). This model belongs to a subclass of GLPV theories with the tensor propagation speed squared $c_{t}^{2}$ equivalent to 1 . The deviation from Horndeski theories is weighed by the dimensionless parameter $\alpha_{\mathrm{H}}=4 x_{4} /\left(5-x_{4}\right)$, where $x_{4}$ is defined in Eq. (3.15). The BH model also has the $a_{2} X^{2}$ and $3 a_{3} X \square \phi$ terms in the Lagrangian, which allow the possibility for approaching a de Sitter attractor from the region $-2<$ $w_{\mathrm{DE}}<-1$ without reaching a tracker solution $\left(w_{\mathrm{DE}}=-2\right)$.

Compared to the standard $\Lambda \mathrm{CDM}$ model, the beyondHorndeski term $x_{4}$ can change the background cosmological dynamics in the early Universe. Since the Hubble expansion rate $H$ is modified by the nonvanishing $x_{4}$ term, this leads to the shift of acoustic peaks of CMB temperature anisotropies at high $\ell$, see BH1 in Fig. 7. Moreover, as we observe in Fig. 4, the early-time dominance of $x_{4}$ over $x_{1,2,3}$ leads to the modified evolution of gravitational potentials $\Psi$ and $\Phi$ in comparison to $\Lambda \mathrm{CDM}$, whose effect is more significant for small-scale perturbations. This modification also affects the evolution of radiation perturbations and the early-time ISW effect. As a result, the amplitude of CMB acoustic peaks is changed by the $x_{4}$ term. These modifications allow us to put bounds on the deviation from Horndeski theories.

The cubic Galileon existing in the BH model leads to the modified growth of matter perturbations and gravitational potentials at low redshifts. Provided that $x_{4}$ is subdominant to $x_{1,2,3}$, the dimensional quantities $\mu$ and $\Sigma$, which characterize the gravitational interactions with matter and light respectively, are given by Eq. (4.6) under the quasistatic approximation deep inside the sound horizon. Thus, the Galileon term $x_{3}$ enhances the linear growth of perturbations without the gravitational slip $(\mu \simeq \Sigma>1)$. This enhancement can be seen in the lensing power spectrum $D_{\ell}^{\phi \phi}$ plotted in Fig. 5.

For the CMB temperature anisotropies, the late-time modified growth of perturbations caused by the cubic Galileon manifests itself in the large-scale ISW tale. The ISW effect is attributed to the variation of the lensing 
gravitational potential $\Psi+\Phi$ related to the quantity $\Sigma$. Unlike the $\Lambda \mathrm{CDM}$ model in which the time derivative $\dot{\Psi}+$ $\dot{\Phi}$ is positive, the Galileon term $x_{3}$ allows the possibility for realizing $\dot{\Psi}+\dot{\Phi}$ closer to 0 . In this case, the large-scale TT power spectrum is lower than that in $\Lambda \mathrm{CDM}$, see GGC and $\mathrm{BH} 2$ in Fig. 7. Moreover, the modified background evolution at low redshifts induced by the Galileon leads to the shift of small-scale CMB acoustic peaks toward higher multipoles. If the contribution of $x_{3}$ to the total dark energy density is increased further, the ISW tale is subject to the significant enhancement compared to $\Lambda \mathrm{CDM}$, together with the large shift of high- $\ell \mathrm{CMB}$ acoustic peaks (see BH3 in Fig. 7). These large modifications to the TT power spectrum also arise for covariant Galileons without the $x_{2}$ term, whose behavior is disfavored from the CMB data $[109,110]$. In the $\mathrm{BH}$ model, the existence of $x_{2}$ besides $x_{3}$ can give rise to the moderately modified TT power spectrum being compatible with the data.

We put observational constraints on free parameters in the $\mathrm{BH}$ model by running the MCMC simulation with the datasets of CMB, BAO, SN Ia, and RSDs. With the Planck CMB data, we showed that today's value of $x_{4}$ is constrained to be smaller than the order $10^{-6}$. Inclusion of other datasets does not modify the order of upper limit of $x_{4}^{(0)}$, and hence $\left|\alpha_{\mathrm{H}}^{(0)}\right| \leq \mathcal{O}\left(10^{-6}\right)$. Apart from the bound arising from the GW decay to dark energy, this is the tightest bound on $\left|\alpha_{\mathrm{H}}^{(0)}\right|$ derived so far from cosmological observations.

The other dark energy density parameters $x_{1}^{(0)}, x_{2}^{(0)}, x_{3}^{(0)}$ are constrained to be in a similar way to those derived in Ref. [71]. The best-fit value of $x_{3}^{(0)}$ is smaller than $\left|x_{1}^{(0)}\right|$ and $x_{2}^{(0)}$ by 1 order of magnitude. This intermediate value of $x_{3}^{(0)}$ leads to the CMB TT power spectrum with modifications at both large and small scales, in such a way that the $\mathrm{BH}$ model can be observationally favored over $\Lambda$ CDM. The evolution of matter perturbations at low redshifts is not subject to the large modification by this intermediate value of $x_{3}^{(0)}$ in comparison to $\Lambda \mathrm{CDM}$, so the $\mathrm{BH}$ model is also compatible with the RSD data. The best-fit background expansion history corresponds to the case in which $w_{\mathrm{DE}}$ finally approaches -1 from the phantom region $-2<w_{\mathrm{DE}}<-1$, whose behavior is consistent with the datasets of SN Ia and BAO. We also showed that, as in the $\Lambda \mathrm{CDM}$ model, the tensions in $H_{0}$ and $\sigma_{8}^{(0)}$ between CMB and low-redshift measurements are not alleviated for the datasets used in our analysis. Future investigations including nonlinear effects and additional probes from weak lensing measurements will allow us to shed light on the possibility for alleviating such tensions in the BH model.

To make comparison between $\mathrm{BH}$ and $\Lambda \mathrm{CDM}$ models, we computed the DIC defined by Eq. (5.3) penalizing complex models with more free parameters. In $\mathrm{BH}$, there are three additional parameters than those in $\Lambda \mathrm{CDM}$. We found that the effective $\chi_{\text {eff }}^{2}$ statistics in $\mathrm{BH}$ is smaller than that in $\Lambda \mathrm{CDM}$ for two combinations of datasets (Planck and PBRS). This is mostly due to both the suppressed ISW tail in $\mathrm{BH}$ and the shifts of hgih- $\ell$ acoustic peaks of the CMB TT power spectrum. These combined effects allow the $\mathrm{BH}$ model to fit the Planck data better. According to the DIC, however, there is a slight preference of $\Lambda \mathrm{CDM}$ over $\mathrm{BH}$ with both Planck and PBRS datasets. The beyond-Horndeski term $x_{4}$ generally works to prevent better fits to the data. The GGC model, which corresponds to $x_{4}=0$ with one parameter less than those in $\mathrm{BH}$, is statistically favored over $\Lambda \mathrm{CDM}$ even with the DIC [71]. This means that, at least in the BH model, there is no preference for the departure from Horndeski theories in cosmological observations.

We have thus shown that the deviation from Horndeski theories is severely constrained by the current observational data, especially from CMB. In spite of this restriction, the best-fit $\mathrm{BH}$ model gives the effective $\chi_{\text {eff }}^{2}$ statistics smaller than that in $\Lambda$ CDM. Moreover, the GGC model with $\alpha_{\mathrm{H}}=0$ leads to the smaller DIC relative to $\Lambda \mathrm{CDM}$, even with two additional parameters. Thus, the BH and GGC models can be compelling and viable candidates for dark energy. Further investigations may be performed in several directions. In this work we considered massless neutrinos, but we plan to extend the analysis to include massive neutrinos and inquire about any degeneracy which can arise between such fluid components and modified gravitational interactions. Moreover, it is of interest to investigate cross-correlations between the ISW signal and galaxy distributions, which can be used to place further constraints on BH and GGC models.

\section{ACKNOWLEDGMENTS}

We thank Matteo Martinelli for support in the numerical implementation. We are grateful to N. Bartolo, A. De Felice, R. Kase, M. Liguori, S. Nakamura, M. Raveri and A. Silvestri for useful discussions and comments. S. P. acknowledges support from the Nederlandse Organisatie voor Wetenschappelijk Onderzoek (NWO) and the Dutch Ministry of Education, Culture and Science (OCW), and also from the Delta Institute for Theoretical Physics (D-ITP) consortium, a program of the NWO that is funded by the OCW. G. B. acknowledges financial sup- port from Fondazione Ing. Aldo Gini. The research of N. F. is supported by Fundação para a Ciência e a Tecnologia (FCT) through national funds (UID/FIS/04434/2013), by FEDER through COMPETE2020 (POCI-01-0145FEDER-007672) and by FCT project "DarkRippleSpacetime ripples in the dark gravitational universe" with Ref. No. PTDC/FIS-OUT/29048/2017. N. F., S. P. and G. B. acknowledge the COST Action (CANTATA/CA15117), supported by COST (European Cooperation in Science and Technology). S. T. is supported by the Grant-in-Aid for Scientific Research Fund of the JSPS No. 19K03854 and MEXT KAKENHI Grant-in-Aid for Scientific Research on Innovative Areas "Cosmic Acceleration" (No. 15H05890). 
[1] S. Weinberg, Rev. Mod. Phys. 61, 1 (1989).

[2] J. Martin, C.R. Phys. 13, 566 (2012).

[3] A. Padilla, arXiv:1502.05296.

[4] A. G. Riess, L. Macri, S. Casertano, H. Lampeitl, H. C. Ferguson, A. V. Filippenko, S. W. Jha, W. Li, and R. Chornock, Astrophys. J. 730, 119 (2011).

[5] T. Delubac et al. (BOSS Collaboration), Astron. Astrophys. 574, A59 (2015).

[6] A. G. Riess et al., Astrophys. J. 826, 56 (2016).

[7] T. M. C. Abbott et al. (DES Collaboration), Mon. Not. R. Astron. Soc. 480, 3879 (2018).

[8] T. M. C. Abbott et al. (DES Collaboration), Phys. Rev. D 98, 043526 (2018).

[9] J. T. A. de Jong et al., Astron. Astrophys. 582, A62 (2015).

[10] K. Kuijken et al., Mon. Not. R. Astron. Soc. 454, 3500 (2015).

[11] H. Hildebrandt et al., Mon. Not. R. Astron. Soc. 465, 1454 (2017).

[12] I. F. Conti, R. Herbonnet, H. Hoekstra, J. Merten, L. Miller, and M. Viola, Mon. Not. R. Astron. Soc. 467, 1627 (2017).

[13] M. Douspis, L. Salvati, and N. Aghanim, Proc. Sci., EDSU2018 (2018) 037.

[14] E. J. Copeland, M. Sami, and S. Tsujikawa, Int. J. Mod. Phys. D 15, 1753 (2006).

[15] A. Silvestri and M. Trodden, Rep. Prog. Phys. 72, 096901 (2009).

[16] S. Tsujikawa, Lect. Notes Phys. 800, 99 (2010).

[17] T. Clifton, P. G. Ferreira, A. Padilla, and C. Skordis, Phys. Rep. 513, 1 (2012).

[18] A. Joyce, B. Jain, J. Khoury, and M. Trodden, Phys. Rep. 568, 1 (2015).

[19] R. Kase and S. Tsujikawa, Int. J. Mod. Phys. D 28, 1942005 (2019).

[20] K. Koyama, Rep. Prog. Phys. 79, 046902 (2016).

[21] Y. Fujii and K. Maeda, The Scalar-Tensor Theory of Gravitation (Cambridge University Press, Cambridge, England, 2003).

[22] G. W. Horndeski, Int. J. Theor. Phys. 10, 363 (1974).

[23] C. Deffayet, X. Gao, D. A. Steer, and G. Zahariade, Phys. Rev. D 84, 064039 (2011).

[24] T. Kobayashi, M. Yamaguchi, and J. Yokoyama, Prog. Theor. Phys. 126, 511 (2011).

[25] C. Charmousis, E. J. Copeland, A. Padilla, and P. M. Saffin, Phys. Rev. Lett. 108, 051101 (2012).

[26] J. Gleyzes, D. Langlois, F. Piazza, and F. Vernizzi, Phys. Rev. Lett. 114, 211101 (2015).

[27] C. Lin, S. Mukohyama, R. Namba, and R. Saitou, J. Cosmol. Astropart. Phys. 10 (2014) 071.

[28] J. Gleyzes, D. Langlois, F. Piazza, and F. Vernizzi, J. Cosmol. Astropart. Phys. 02 (2015) 018.

[29] L. A. Gergely and S. Tsujikawa, Phys. Rev. D 89, 064059 (2014).

[30] R. Kase and S. Tsujikawa, Phys. Rev. D 90, 044073 (2014).

[31] A. De Felice, K. Koyama, and S. Tsujikawa, J. Cosmol. Astropart. Phys. 05 (2015) 058.

[32] A. De Felice, N. Frusciante, and G. Papadomanolakis, J. Cosmol. Astropart. Phys. 03 (2017) 027.

[33] T. Kobayashi, Y. Watanabe, and D. Yamauchi, Phys. Rev. D 91, 064013 (2015).
[34] K. Koyama and J. Sakstein, Phys. Rev. D 91, 124066 (2015).

[35] J. Sakstein, Phys. Rev. Lett. 115, 201101 (2015).

[36] J. Sakstein, Phys. Rev. D 92, 124045 (2015).

[37] J. Sakstein, H. Wilcox, D. Bacon, K. Koyama, and R. C. Nichol, J. Cosmol. Astropart. Phys. 07 (2016) 019.

[38] E. Babichev, K. Koyama, D. Langlois, R. Saito, and J. Sakstein, Classical Quantum Gravity 33, 235014 (2016).

[39] A. De Felice, R. Kase, and S. Tsujikawa, Phys. Rev. D 92, 124060 (2015).

[40] R. Kase, S. Tsujikawa, and A. De Felice, J. Cosmol. Astropart. Phys. 03 (2016) 003.

[41] X. Gao, Phys. Rev. D 90, 104033 (2014).

[42] R. Kase and S. Tsujikawa, Int. J. Mod. Phys. D 23, 1443008 (2014).

[43] N. Frusciante, M. Raveri, D. Vernieri, B. Hu, and A. Silvestri, Phys. Dark Universe 13, 7 (2016).

[44] P. Horava, Phys. Rev. D 79, 084008 (2009).

[45] D. Langlois and K. Noui, J. Cosmol. Astropart. Phys. 02 (2016) 034.

[46] D. Langlois and K. Noui, J. Cosmol. Astropart. Phys. 07 (2016) 016.

[47] M. Crisostomi, K. Koyama, and G. Tasinato, J. Cosmol. Astropart. Phys. 04 (2016) 044.

[48] H. Motohashi, K. Noui, T. Suyama, M. Yamaguchi, and D. Langlois, J. Cosmol. Astropart. Phys. 07 (2016) 033.

[49] B. P. Abbott et al. (LIGO Scientific and Virgo Collaborations), Phys. Rev. Lett. 119, 161101 (2017).

[50] A. Goldstein et al., Astrophys. J. 848, L14 (2017).

[51] B. P. Abbott et al. (LIGO Scientific and Virgo and FermiGBM and INTEGRAL Collaborations), Astrophys. J. 848, L13 (2017).

[52] P. Creminelli and F. Vernizzi, Phys. Rev. Lett. 119, 251302 (2017).

[53] J. M. Ezquiaga and M. Zumalacarregui, Phys. Rev. Lett. 119, 251304 (2017).

[54] J. Sakstein and B. Jain, Phys. Rev. Lett. 119, 251303 (2017).

[55] T. Baker, E. Bellini, P. G. Ferreira, M. Lagos, J. Noller, and I. Sawicki, Phys. Rev. Lett. 119, 251301 (2017).

[56] L. Amendola, M. Kunz, I. D. Saltas, and I. Sawicki, Phys. Rev. Lett. 120, 131101 (2018).

[57] L. Amendola, M. Kunz, I. D. Saltas, and I. Sawicki, Phys. Rev. Lett. 120, 131101 (2018).

[58] R. A. Battye, F. Pace, and D. Trinh, Phys. Rev. D 98, 023504 (2018).

[59] L. Amendola, D. Bettoni, G. Domenech, and A. R. Gomes, J. Cosmol. Astropart. Phys. 06 (2018) 029.

[60] E. J. Copeland, M. Kopp, A. Padilla, P. M. Saffin, and C. Skordis, Phys. Rev. Lett. 122, 061301 (2019).

[61] R. Kase and S. Tsujikawa, Phys. Rev. D 97, 103501 (2018).

[62] P. Creminelli, M. Lewandowski, G. Tambalo, and F. Vernizzi, J. Cosmol. Astropart. Phys. 12 (2018) 025.

[63] N. Frusciante, R. Kase, K. Koyama, S. Tsujikawa, and D. Vernieri, Phys. Lett. B 790, 167 (2019).

[64] T. Kobayashi, Rep. Prog. Phys. 82, 086901 (2019).

[65] C. de Rham and S. Melville, Phys. Rev. Lett. 121, 221101 (2018).

[66] H. Audley et al. (LISA Collaboration), arXiv:1702.00786. 
[67] R. K. Jain, C. Kouvaris, and N. G. Nielsen, Phys. Rev. Lett. 116, 151103 (2016).

[68] I. D. Saltas, I. Sawicki, and I. Lopes, J. Cosmol. Astropart. Phys. 05 (2018) 028.

[69] A. Dima and F. Vernizzi, Phys. Rev. D 97, 101302 (2018).

[70] D. Traykova, E. Bellini, and P. G. Ferreira, arXiv:1902 .10687.

[71] S. Peirone, G. Benevento, N. Frusciante, and S. Tsujikawa, arXiv:1905.05166.

[72] B. Hu, M. Raveri, N. Frusciante, and A. Silvestri, Phys. Rev. D 89, 103530 (2014).

[73] M. Raveri, B. Hu, N. Frusciante, and A. Silvestri, Phys. Rev. D 90, 043513 (2014).

[74] A. Nicolis, R. Rattazzi, and E. Trincherini, Phys. Rev. D 79, 064036 (2009).

[75] C. Deffayet, G. Esposito-Farese, and A. Vikman, Phys. Rev. D 79, 084003 (2009).

[76] N. Arkani-Hamed, H. C. Cheng, M. A. Luty, and S. Mukohyama, J. High Energy Phys. 05 (2004) 074.

[77] A. De Felice and S. Tsujikawa, Phys. Rev. Lett. 105, 111301 (2010).

[78] S. Nesseris, A. De Felice, and S. Tsujikawa, Phys. Rev. D 82, 124054 (2010).

[79] N. Bartolo, E. Bellini, D. Bertacca, and S. Matarrese, J. Cosmol. Astropart. Phys. 03 (2013) 034.

[80] G. Gubitosi, F. Piazza, and F. Vernizzi, J. Cosmol. Astropart. Phys. 02 (2013) 032.

[81] J. K. Bloomfield, E. E. Flanagan, M. Park, and S. Watson, J. Cosmol. Astropart. Phys. 08 (2013) 010.

[82] J. Bloomfield, J. Cosmol. Astropart. Phys. 12 (2013) 044.

[83] J. Gleyzes, D. Langlois, F. Piazza, and F. Vernizzi, J. Cosmol. Astropart. Phys. 08 (2013) 025.

[84] J. Gleyzes, D. Langlois, and F. Vernizzi, Int. J. Mod. Phys. D 23, 1443010 (2014).

[85] A. Lewis, A. Challinor, and A. Lasenby, Astrophys. J. 538, 473 (2000).

[86] A. Lewis and S. Bridle, Phys. Rev. D 66, 103511 (2002).

[87] R. L. Arnowitt, S. Deser, and C. W. Misner, Gen. Relativ. Gravit. 40, 1997 (2008).

[88] N. Frusciante, G. Papadomanolakis, and A. Silvestri, J. Cosmol. Astropart. Phys. 07 (2016) 018.

[89] N. Frusciante, S. Peirone, S. Casas, and N. A. Lima, Phys. Rev. D 99, 063538 (2019).
[90] L. Amendola, M. Kunz, and D. Sapone, J. Cosmol. Astropart. Phys. 04 (2008) 013.

[91] E. Bertschinger and P. Zukin, Phys. Rev. D 78, 024015 (2008).

[92] L. Pogosian, A. Silvestri, K. Koyama, and G. B. Zhao, Phys. Rev. D 81, 104023 (2010).

[93] B. Boisseau, G. Esposito-Farese, D. Polarski, and A. A. Starobinsky, Phys. Rev. Lett. 85, 2236 (2000).

[94] S. Tsujikawa, Phys. Rev. D 76, 023514 (2007).

[95] A. De Felice, T. Kobayashi, and S. Tsujikawa, Phys. Lett. B 706, 123 (2011).

[96] S. Tsujikawa, Phys. Rev. D 92, 044029 (2015).

[97] S. Peirone, K. Koyama, L. Pogosian, M. Raveri, and A. Silvestri, Phys. Rev. D 97, 043519 (2018).

[98] P. A. R. Ade et al. (Planck Collaboration), Astron. Astrophys. 594, A13 (2016).

[99] A. Lewis and A. Challinor, Phys. Rep. 429, 1 (2006).

[100] U. Seljak and M. Zaldarriaga, Astrophys. J. 469, 437 (1996).

[101] M. X. Lin, M. Raveri, and W. Hu, Phys. Rev. D 99, 043514 (2019).

[102] G. Benevento, M. Raveri, A. Lazanu, N. Bartolo, M. Liguori, P. Brax, and P. Valageas, J. Cosmol. Astropart. Phys. 05 (2019) 027.

[103] W. Hu and N. Sugiyama, Astrophys. J. 444, 489 (1995).

[104] N. Aghanim et al. (Planck Collaboration), Astron. Astrophys. 594, A11 (2016).

[105] F. Beutler, C. Blake, M. Colless, D. H. Jones, L. StaveleySmith, L. Campbell, Q. Parker, W. Saunders, and F. Watson, Mon. Not. R. Astron. Soc. 416, 3017 (2011).

[106] A. J. Ross, L. Samushia, C. Howlett, W. J. Percival, A. Burden, and M. Manera, Mon. Not. R. Astron. Soc. 449, 835 (2015).

[107] S. Alam et al. (BOSS Collaboration), Mon. Not. R. Astron. Soc. 470, 2617 (2017).

[108] M. Betoule et al. (SDSS Collaboration), Astron. Astrophys. 568, A22 (2014).

[109] J. Renk, M. Zumalacaregui, F. Montanari, and A. Barreira, J. Cosmol. Astropart. Phys. 10 (2017) 020.

[110] S. Peirone, N. Frusciante, B. Hu, M. Raveri, and A. Silvestri, Phys. Rev. D 97, 063518 (2018).

[111] A. G. Riess et al., Astrophys. J. 855, 136 (2018).

[112] D. J. Spiegelhalter, N. G. Best, B. P. Carlin, and A. van der Linde, J. R. Stat. Soc. Ser. B 76, 485 (2014). 